\title{
Hyperequivalence of Logic Programs with Respect to Supported Models*
}

\author{
Mirosław Truszczyński \\ Department of Computer Science \\ University of Kentucky \\ Lexington, KY 40506-0046, USA \\ mirek@cs.uky.edu
}

\author{
Stefan Woltran \\ Institut für Informationssysteme 184/2 \\ Technische Universität Wien \\ Favoritenstraße 9-11 \\ A-1040 Vienna, Austria \\ woltran@dbai.tuwien.ac.at
}

Dedicated to Victor Marek

\begin{abstract}
Recent research in nonmonotonic logic programming has focused on certain types of program equivalence, which we refer to here as hyperequivalence, that are relevant for program optimization and modular programming. So far, most results concern hyperequivalence relative to the stable-model semantics. However, other semantics for logic programs are also of interest, especially the semantics of supported models which, when properly generalized, is closely related to the autoepistemic logic of Moore. In this paper, we consider a family of hyperequivalence relations for programs based on the semantics of supported and supported minimal models. We characterize these relations in model-theoretic terms. We use the characterizations to derive complexity results concerning testing whether two programs are hyperequivalent relative to supported and supported minimal models.
\end{abstract}

\section{Introduction}

The problem of the equivalence of logic programs with respect to the stable-model semantics has received substantial attention in the answer-set programming research community in the past several years (Lifschitz, Pearce, \& Valverde 2001; Lin 2002; Turner 2003; de Jongh \& Hendriks 2003; Inoue \& Sakama 2004; Eiter, Tompits, \& Woltran 2005; Ferraris 2005; Eiter, Fink, \& Woltran 2007; Oikarinen \& Janhunen 2006; Lin \& Chen 2007; Oetsch, Tompits, \& Woltran 2007; Woltran 2008; Wong 2008; Gebser et al. 2008). The problem can be stated as follows. Let $\mathcal{C}$ be a class of logic programs containing the empty program. We say that programs $P$ and $Q$ are equivalent with respect to $\mathcal{C}$ if for every program $R \in \mathcal{C}, P \cup R$ and $Q \cup R$ have the same stable models. We refer to programs in $\mathcal{C}$ as contexts. Clearly, as the empty program is always assumed to be one of the context programs, the equivalence with respect to $\mathcal{C}$ implies the standard nonmonotonic equivalence of programs, where two programs $P$ and $Q$ are nonmonotonically

${ }^{*}$ This work was partially supported by the NSF grant IIS0325063, the KSEF grant KSEF-1036-RDE-008, and by the Austrian Science Fund (FWF) under grants P18019-N04 and P20704N18.

Copyright (c) 2008, authors listed above. All rights reserved. equivalent if they have the same stable models. Therefore, we will refer to these stronger versions of equivalence collectively as hyperequivalence.

Understanding hyperequivalence is fundamental for the development of modular answer-set programs and knowledge bases. The problem is non-trivial due to the nonmonotonic nature of the stable-model semantics. If $S$ is a module within a larger program $T$, replacing $S$ with $S^{\prime}$ results in the program $T^{\prime}=(T \backslash S) \cup S^{\prime}$, which must have the same meaning (the same stable models) as $T$. The nonmonotonic equivalence of $S$ and $S^{\prime}$ does not guarantee it. The hyperequivalence of $S$ and $S^{\prime}$ relative to the class of all programs does. However, the latter may be too restrictive an approach in certain application scenarios, in particular if properties of possible instantiations of $T$ are known in advance.

Thus, several notions of hyperequivalence that impose restrictions on the context class $\mathcal{C}$ have been studied. If $\mathcal{C}$ is unrestricted, that is, any program is a possible context, we obtain strong equivalence (Lifschitz, Pearce, \& Valverde 2001). If $\mathcal{C}$ is the collection of all sets of facts, we obtain uniform equivalence (Eiter, Fink, \& Woltran 2007). Another direction is to restrict the alphabet over which contexts are given. The resulting notions of hyperequivalence are called relativized (with respect to the context alphabet), and can be combined with strong and uniformly equivalence (Eiter, Fink, \& Woltran 2007). Even more generally, we can specify different alphabets for bodies and heads of rules in contexts. This gives rise to a unifying view on strong and uniform equivalence (Woltran 2008). A yet different approach to hyperequivalence is to compare only some dedicated projected output atoms rather than entire stable models (Eiter, Tompits, \& Woltran 2005; Oikarinen \& Janhunen 2006; Oetsch, Tompits, \& Woltran 2007).

All those results concern hyperequivalence with respect to the stable-model semantics of programs. There has been little work on other semantics, with (Cabalar et al. 2006) being a notable exception. In this paper, we address the problem of the hyperequivalence with respect to two other major semantics of programs, those of supported models (Clark 1978) and supported minimal models.

We are motivated in our studies by the role both semantics play in logic programing and, more generally, in nonmonotonic reasoning. The supported model semantics was the first major step leading to a formal account of default 
negation operator in logic programming and a springboard towards the stable-model semantics. Through the notions of program completion (Clark 1978) and loop formula (Lin \& Zhao 2002), it links stable models of programs with models of propositional theories and facilitates the use of SAT solvers to compute stable models. The link is especially direct for the class of tight programs, for which supported and stable models coincide. Next, logic programming with the semantics of supported models, under the interpretation proposed by Konolige (Konolige 1988; 1989), forms a significant fragment of the autoepistemic logic (Moore 1985) - one of the key formalisms of nonmonotonic reasoning.

Since the minimality property is fundamental from the perspective of knowledge representation, we also consider in the paper the semantics of supported minimal models, that is, supported models that are minimal as models. While it seems to have received little attention in the area of logic programming, it has been studied extensively in a more general setting of modal nonmonotonic logics, first under the name of the semantics of moderately grounded expansions for autoepistemic logic (Konolige 1988; 1989) and then, under the name of ground $\mathcal{S}$-expansions, for an arbitrary nonmonotonic modal logic $\mathcal{S}$ (Kaminski 1991; Truszczyński 1991). The complexity of reasoning with moderately grounded expansions was established in (Eiter $\&$ Gottlob 1992) to be complete for classes at the third level of the polynomial hierarchy. As before, under the interpretation of Konolige, logic programming with the semantics of supported minimal models forms an important fragment of autoepistemic logic with the semantics of moderately grounded expansions.

In the paper, we define several concepts of hyperequivalence, depending on the class of programs allowed as contexts. We obtain characterizations of hyperequivalence with respect to supported (minimal) models in terms of semantic objects, similar to SE-models (Turner 2003) or UE-models (Eiter, Fink, \& Woltran 2007), that one can attribute to programs.

The characterizations allow us to derive results on the complexity of problems to decide whether two programs are hyperequivalent with respect to supported (minimal) models. They are especially useful in establishing upper bounds which, typically, are easy to derive but in the context of hyperequivalence are not obvious. Our results paint a detailed picture of the complexity landscape for relativized hyperequivalence with respect to supported (minimal) models.

The impact of our results goes beyond logic programming with the semantics of supported and supported models. For tight programs, they imply new characterizations of certain notions of hyperequivalence with respect to stable-model semantics. They also yield characterizations of hyperequivalence of some autoepistemic theories with respect to the semantics of expansions and moderately grounded expansions, that can be derived based on the Konolige's interpretation of programs as modal (autoepistemic) theories.

\section{Preliminaries}

We fix a countable set $A t$ of atoms (possibly infinite). All programs we consider here consist of rules of the form

$$
a_{1}|\ldots| a_{k} \leftarrow b_{1}, \ldots, b_{m}, \operatorname{not} c_{1}, \ldots, \operatorname{not} c_{n},
$$

where $a_{i}, b_{i}$ and $c_{i}$ are atoms in $A t$, '।' stands for the disjunction, ', stands for the conjunction, and not is the default negation. If $k=0$, the rule is a constraint. If $k \leq 1$, the rule is normal.

For a rule $r$ of the form given above, we call the set $\left\{a_{1}, \ldots, a_{k}\right\}$ the head of $r$ and denote it by $h d(r)$. Similarly, we call the conjunction $b_{1}, \ldots, b_{m}$, not $c_{1}, \ldots$, not $c_{n}$ the body of $r$ and denote it by $b d(r)$. We also write $b d^{+}(r)=$ $\left\{b_{1}, \ldots, b_{m}\right\}$ and $b d^{-}(r)=\left\{c_{1}, \ldots, c_{n}\right\}$, and denote by $b d^{ \pm}(r)$ the set of all atoms occurring in the body of $r$, that is, $b d^{ \pm}(r)=b d^{+}(r) \cup b d^{-}(r)$. Moreover, for a program $P$, we set $h d(P)=\bigcup_{r \in P} h d(r)$, and $b d^{ \pm}(P)=\bigcup_{r \in P} b d^{ \pm}(r)$.

An interpretation $M \subseteq A t$ is a model of a rule $r$, written $M \models r$, if whenever $M$ satisfies every literal in $b d(r)$, written $M \models b d(r)$, we have that $h d(r) \cap M \neq \emptyset$, written $M \models h d(r)$.

An interpretation $M \subseteq A t$ is a model of a program $P$, written $M \models P$, if $M \models r$ for every $r \in P$. If, in addition, $M$ is a minimal hitting set of $\{h d(r) \mid r \in P$ and $M \models$ $b d(r)\}$, then $M$ is a supported model of $P$ (Brass \& Dix 1997; Inoue \& Sakama 1998). It is well known that $M \subseteq A t$ is a supported model of $P$ if and only if $M$ is a model of $P$ and for every $a \in M$ there is a rule $r \in P$ such that $M \models b d(r)$ and $\{a\}=h d(r) \cap M$. We say that each such rule $r$ supports $a$ with respect to $M$.

For a rule $r=a_{1}|\ldots| a_{k} \leftarrow b d$, where $k \geq 1$, a shift of $r$ is a normal program rule of the form

$$
a_{i} \leftarrow b d, n o t a_{1}, \ldots, n o t a_{i-1}, n o t a_{i+1}, \ldots, n o t a_{k},
$$

where $i=1, \ldots, k$. If $r$ is a normal rule, the only shift of $r$ is $r$ itself. A program consisting of all shifts of rules in a program $P$ is the shift of $P$. We denote it by $\operatorname{sh}(P)$. It is evident that a set $Y$ of atoms is a (minimal) model of $P$ if and only if $Y$ is a (minimal) model of $\operatorname{sh}(P)$. It is also easy to check that $Y$ is a supported model of $P$ if and only if it is a supported model of $\operatorname{sh}(P)$.

Supported models of a normal logic program $P$ have a useful characterization in terms of the (partial) one-step provability operator $T_{P}$ (van Emden \& Kowalski 1976), defined as follows. For $M \subseteq A t$, if there is a constraint $r \in P$ such that $M \models b d(r)$ (that is, $M \forall r$ ), then $T_{P}(M)$ is undefined. Otherwise,

$$
T_{P}(M)=\{h d(r) \mid r \in P \text { and } M \models b d(r)\} .
$$

Whenever we use $T_{P}(M)$ in a relation such as (proper) inclusion, equality or inequality, we always implicitly assume that $T_{P}(M)$ is defined.

It is well known that $M$ is a model of $P$ if and only if $T_{P}(M) \subseteq M$ (that is, $T_{P}$ is defined for $M$ and satisfies $\left.T_{P}(M) \subseteq M\right)$. Similarly, $M$ is a supported model of $P$ if $T_{P}(M)=M$ (that is, $T_{P}$ is defined for $M$ and satisfies $\left.T_{P}(M)=M\right)($ Apt 1990). 
It follows that $M$ is a model of a disjunctive program $P$ if and only if $T_{s h(P)}(M) \subseteq M$. Moreover, $M$ is a supported model of $P$ if and only if $T_{s h(P)}(M)=M$.

In the paper we will also consider an important variant of the semantics of supported models, combining it with the principle of minimality. A set $M$ of atoms is a supported minimal model (suppmin model, for short) of a logic program $P$ if it is a supported model of $P$ and a minimal model of $P$.

To illustrate the relationships between the semantics, we recall that stable models are suppmin models. Moreover, directly from the definition we have that suppmin models are supported models. Lastly, supported models are models. However, these implications cannot be reversed as demonstrated by the following example.

Example 2.1 Let $P=\{a \leftarrow a\}$. Then every interpretation is a model of $P$. One can check that $T_{P}(\emptyset)=\emptyset$ and $T_{P}(\{a\})=\{a\}$. Thus, $\emptyset$ and $\{a\}$ are supported models of $P$. In fact, they are the only supported models of P. Moreover, $\emptyset$ is the only stable and also the only suppmin model of $P$.

Next, let $Q=\{a \leftarrow b ; b \leftarrow a ; \leftarrow$ not $a\}$. Then $Q$ has no stable models but $\{a, b\}$ is a suppmin model of $Q$.

\section{Hyperequivalence with respect to supported models}

Let $\mathcal{C}$ be a class of programs (contexts) containing the empty program, the assumption we adopt throughout the paper. Two disjunctive logic programs $P$ and $Q$ are suppequivalent relative to $\mathcal{C}$ if for every $R \in \mathcal{C}, P \cup R$ and $Q \cup R$ have the same supported models. Since $\emptyset \in \mathcal{C}$, if programs $P$ and $Q$ are supp-equivalent relative to $\mathcal{C}$, they have the same supported models. In other words, supp-equivalence implies standard equivalence with respect to supported models.

Supp-equivalence is a non-trivial concept, different than equivalence with respect to models, supported models, stable models, and different than hyperequivalence with respect to stable models.

Example 3.1 Let $P_{0}=\{a\}$ and $Q_{0}=\{a \leftarrow$ not $b\}$. Then $P_{0}$ and $Q_{0}$ have the same supported models $(\{a\}$ is the unique supported model of each program), that is, they are equivalent with respect to supported models. However, $P_{0} \cup\{b\}$ and $Q_{0} \cup\{b\}$ have different supported models $(\{b\}$ and $\{a, b\}$, respectively). Thus, $P_{0}$ and $Q_{0}$ are not suppequivalent relative to any class $\mathcal{C}$ containing $\{b\}$. It follows that the two variants of equivalence are different (but, as we just noted above, our restriction on $\mathcal{C}$ guarantees that suppequivalence implies equivalence with respect to supported models).

Next, let $P_{1}=\{a \leftarrow a\}$ and $Q_{1}=\emptyset$. Clearly, $P_{1}$ and $Q_{1}$ have the same models and the same stable models. Moreover, for every program $R, P_{1} \cup R$ and $Q_{1} \cup R$ have the same stable models, that is, $P_{1}$ and $Q_{1}$ are strongly (and so, also uniformly) equivalent with respect to stable models. However, $P_{1}$ and $Q_{1}$ have different supported models. Thus, they are not supp-equivalent relative to any class of programs that contains the empty program.
Next, let $P_{2}=\{a \leftarrow a ; a \leftarrow$ not $a\}$ and $Q_{2}=\{a\}$. One can check that for every program $R, P_{2} \cup R$ and $Q_{2} \cup R$ have the same supported models, that is, $P_{2}$ and $Q_{2}$ are suppequivalent relative to any class of programs. However, $P_{2}$ and $Q_{2}$ do not have the same stable models and so, they are not equivalent with respect to stable models nor hyperequivalent with respect to stable models relative to any class of programs (containing the empty program).

Finally, let $P_{3}=\{\leftarrow b\} \cup P_{2}$ and $Q_{3}=Q_{2}$. Then, $P_{3}$ and $Q_{3}$ are neither hyperequivalent with respect to stable models relative to any class of programs nor equivalent with respect to classical models. However, for any program $R$ such that $b$ does not appear in rule heads of $R, P_{3} \cup R$ and $Q_{3} \cup R$ have the same supported models, that is, $P_{3}$ and $Q_{3}$ are supp-equivalent with respect to each such class of programs (we will verify this claim independently later by using our characterization of supp-equivalence).

As we will see, supp-equivalence with respect to all programs implies equivalence with respect to models and so, it is not a coincidence that in the last example we used a restricted class of contexts. To see that $P_{3}$ and $Q_{3}$ are not supp-equivalent with respect to the class of all programs, one can consider $R=\{b\}$. Then, $\{a, b\}$ is a supported model of $Q_{3} \cup R$, but not of $P_{3} \cup R$.

We observe that supp-equivalence relative to $\mathcal{C}$ implies supp-equivalence relative to any $\mathcal{C}^{\prime}$, such that $\mathcal{C}^{\prime} \subseteq \mathcal{C}$, but the converse is not true in general as illustrated by programs $P_{3}$ and $Q_{3}$.

In this section we characterize supp-equivalence relative to classes of programs defined in terms of atoms that can appear in the heads and in the bodies of rules. Let $A, B \subseteq A t$. By $\mathcal{H B}^{d}(A, B)$ we denote the class of all disjunctive programs $P$ such that $h d(P) \subseteq A$ (atoms in the heads of rules in $P$ must be from $A$ ) and $b d^{ \pm}(P) \subseteq B$ (atoms in the bodies of rules in $P$ must be from $B$ ). We denote by $\mathcal{H B}^{n}(A, B)$ the class of all normal programs in $\mathcal{H B}^{d}(A, B)$ (possibly with constraints). These classes of programs were considered in the context of hyperequivalence of programs with respect to the stable-model semantics in (Woltran 2008).

We focus first on the case when programs compared for equivalence, as well as the contexts, are normal. The restriction will allow us to take advantage of the one-step provability operator. Later, we will obtain a characterization of supp-equivalence for the general disjunctive case as a corollary.

Given a normal program $P$, and a set $A \subseteq A t$, we define

$$
\operatorname{Mod}_{A}(P)=\left\{Y \subseteq A t \mid Y \models P \text { and } Y \backslash T_{P}(Y) \subseteq A\right\} .
$$

We call interpretations in $\operatorname{Mod}_{A}(P) A$-models of $P$. An $A$-model $Y$ of $P$ can be viewed as a candidate for becoming a supported model of an extension of $P$ with some program $R \in \mathcal{H B}^{n}(A, B)$. Indeed, such a candidate set has to be classical model of $P$ (otherwise it cannot be a supported model, no matter how $P$ is extended). Moreover, the elements from $Y \backslash T_{P}(Y)$ have to be contained in $A$. Otherwise programs from $\mathcal{H} \mathcal{B}^{n}(A, B)$ cannot close this gap. Intuitively, if there is an $A$-model of a program $P$ that is not an $A$-model of a program $Q$, we are able to find a context 
$R \in \mathcal{H B}^{n}(A, B)$ such that $Y$ is a supported model of $P \cup R$ but not of $Q \cup R$. Thus, $P$ and $Q$ cannot be supp-equivalent relative to $\mathcal{H B}^{n}(A, B)$. Similarly, if $P$ and $Q$ have the same $A$-models but, for one of them, say $Y, T_{P}(Y) \neq T_{Q}(Y)$, then again it is possible to extend $P$ and $Q$ by a context $R \in \mathcal{H B}^{n}(A, B)$ so that $Y$ is a supported model of one of these programs only. Thus, having the same $A$-models, and revising each $A$-model in the same way by means of the one-step provability operator are two necessary conditions for supp-equivalence with respect to $\mathcal{H} \mathcal{B}^{n}(A, B)$. It turns out that the two conditions together are also sufficient. More precisely, we have the following characterization of the supp-equivalence relative to $\mathcal{H B}^{n}(A, B)$.

Theorem 3.2 Let $P$ and $Q$ be normal programs, $A \subseteq A t$, and $\mathcal{C}$ a class of programs such that $\mathcal{H B}^{n}(A, \emptyset) \subseteq \mathcal{C} \subseteq$ $\mathcal{H B}^{n}(A, A t)$. Then, $P$ and $Q$ are supp-equivalent relative to $\mathcal{C}$ if and only if $\operatorname{Mod}_{A}(P)=\operatorname{Mod}_{A}(Q)$ and for every $Y \in \operatorname{Mod}_{A}(P), T_{P}(Y)=T_{Q}(Y)$.

Proof. $(\Rightarrow)$ Since $\mathcal{H} \mathcal{B}^{n}(A, \emptyset) \subseteq \mathcal{C}, P$ and $Q$ are suppequivalent relative to $\mathcal{H} \mathcal{B}^{n}(A, \emptyset)$.

Let $Y \in \operatorname{Mod}_{A}(P)$. It follows that $Y \models P$ and $Y \backslash$ $T_{P}(Y) \subseteq A$. Let us consider $P \cup\left(Y \backslash T_{P}(Y)\right)$ Then

$$
T_{P \cup\left(Y \backslash T_{P}(Y)\right)}(Y)=T_{P}(Y) \cup\left(Y \backslash T_{P}(Y)\right) .
$$

Since $Y \models P, T_{P}(Y) \subseteq Y$. Hence, $T_{P \cup\left(Y \backslash T_{P}(Y)\right)}(Y)=$ $Y$. It follows that $Y$ is a supported model of $P \cup\left(Y \backslash T_{P}(Y)\right)$. Since $Y \backslash T_{P}(Y) \subseteq A, Y \backslash T_{P}(Y) \in \mathcal{H B}^{n}(A, \emptyset)$. Thus, $Y$ is a supported model of $Q \cup\left(Y \backslash T_{P}(Y)\right)$ and, consequently,

$$
Y=T_{Q \cup\left(Y \backslash T_{P}(Y)\right)}(Y)=T_{Q}(Y) \cup\left(Y \backslash T_{P}(Y)\right) .
$$

It follows that $T_{Q}(Y) \subseteq Y$ and $T_{P}(Y) \subseteq T_{Q}(Y)$. Thus, $Y \backslash T_{Q}(Y) \subseteq Y \backslash T_{P}(Y) \subseteq A$ and so, $Y \in \operatorname{Mod}_{A}(Q)$. The converse inclusion follows by the symmetry argument and so, we have $\operatorname{Mod}_{A}(P)=\operatorname{Mod}_{A}(Q)$.

Next, let $Y \in \operatorname{Mod}_{A}(P)$ (and so, $Y \in \operatorname{Mod}_{A}(Q)$, too). We have seen that $T_{P}(Y) \subseteq T_{Q}(Y)$. By the symmetry, $T_{Q}(Y) \subseteq T_{P}(Y)$. Thus, $T_{P}(Y)=T_{Q}(Y)$.

$(\Leftarrow)$ Let $R$ be a logic program from $\mathcal{C}$ and $Y$ be a supported model of $P \cup R$. It follows that $Y=T_{P \cup R}(Y)=$ $T_{P}(Y) \cup T_{R}(Y)$. Thus, $T_{P}(Y) \subseteq Y$ (that is, $Y \models P$ ) and $Y \backslash T_{P}(Y) \subseteq A$ (because $h \bar{d}(R) \subseteq A$ ). We obtain $Y \in \operatorname{Mod}_{A}(P)$ and, by the assumption, $\bar{T}_{Q}(Y)=T_{P}(Y)$. Hence, $Y=T_{Q}(Y) \cup T_{R}(Y)=T_{Q \cup R}(Y)$. That is, $Y$ is a supported model of $Q \cup R$.

We note that our characterization for supp-equivalence relative to $\mathcal{H B}^{n}(A, B)$ does not depend on the bodyalphabet $B$ of the context. Thus, Theorem 3.2 applies, in particular, to $\mathcal{C}=\mathcal{H} \mathcal{B}^{n}(A t, \emptyset)$ and $\mathcal{C}=\mathcal{H} \mathcal{B}^{n}(A t, A t)$. Consequently, it characterizes strong and uniform suppequivalence of normal programs. It also has several corollaries concerned with special cases for $A$. The first one deals with the case when $A=A t$, in which the characterizing condition simplifies.

Corollary 3.3 Let $P$ and $Q$ be normal programs and $\mathcal{C}$ a class of programs such that $\mathcal{H B}^{n}(A t, \emptyset) \subseteq \mathcal{C} \subseteq$ $\mathcal{H B}^{n}(A t, A t)$. Then, $P$ and $Q$ are supp-equivalent relative to $\mathcal{C}$ if and only if $P$ and $Q$ have the same models and for every model $Y$ of $P, T_{P}(Y)=T_{Q}(Y)$.
Proof. When $A=A t, \operatorname{Mod}_{A}(P)$ and $\operatorname{Mod}_{A}(Q)$ consist of models of $P$ and $Q$, respectively. Thus, the result follows directly from Theorem 3.2.

At the other extreme, we have the case $A=\emptyset$. In that case, all context programs consist of constraints (rules with the empty head) only.

Corollary 3.4 Let $P$ and $Q$ be normal programs and $\mathcal{C}$ a class of programs such that $\mathcal{H B}^{n}(\emptyset, \emptyset) \subseteq \mathcal{C} \subseteq \mathcal{H B}^{n}(\emptyset, A t)$. The following conditions are equivalent:

1. $P$ and $Q$ are supp-equivalent relative to $\mathcal{C}$

2. $P$ and $Q$ have the same supported models

3. $\operatorname{Mod}_{\emptyset}(P)=\operatorname{Mod}_{\emptyset}(Q)$.

Proof. [(1) implies (2)]: Since $\emptyset \in \mathcal{H B}^{n}(\emptyset, \emptyset)$, the assertion is obvious.

[(2) implies (3)]: Let $Y \in \operatorname{Mod}_{\emptyset}(P)$. Then, $Y \models P$, that is, $T_{P}(Y) \subseteq Y$, and $Y \backslash T_{P}(Y)=\emptyset$. Thus, $Y=T_{P}(Y)$ and, consequently, $Y$ is a supported model of $P$. By the assumption, $Y$ is a supported model of $Q$, that is $Y=T_{Q}(Y)$. It follows that $Y \in \operatorname{Mod}_{\emptyset}(Q)$. The converse inclusion follows by the symmetry argument.

[(3) implies (1)]: Let $R \in \mathcal{C}$ and let $Y$ be a supported model of $P \cup R$. Then $Y \models P \cup R$ and $Y=T_{P \cup R}(Y)=T_{P}(Y) \cup$ $T_{R}(Y)=T_{P}(Y)$ (indeed, as $Y \models R$ and every rule in $R$ is a constraint, $\left.T_{R}(Y)=\emptyset\right)$. Thus $Y \in \operatorname{Mod}_{\emptyset}(P)$ and so, also $Y \in \operatorname{Mod}_{\emptyset}(Q)$. From the latter we obtain $Y=T_{Q}(Y)$. Since $T_{R}(Y)=\emptyset, Y=T_{Q}(Y) \cup T_{R}(Y)=T_{Q \cup R}(Y)$, that is, $Y$ is a supported model of $Q \cup R$. Again, the other implication follows by the symmetry argument.

We will now apply our results to some pairs of programs discussed in Example 3.1.

Example 3.5 First, we note that $P_{1}$ and $Q_{1}$ have the same models. In particular, $\{a\}$ is a model of both programs. However, $T_{P_{1}}(\{a\})=\{a\}$ and $T_{Q_{1}}(\{a\})=\emptyset$. Thus, $T_{P_{1}}(\{a\}) \neq T_{Q_{1}}(\{a\})$ and so, $P_{1}$ and $Q_{1}$ are not suppequivalent relative to $\mathcal{H B}^{n}(A t, \emptyset)$ (by Corollary 3.3).

On the other hand, $P_{2}$ and $Q_{2}$ have the same models and for every $Y$ (in particular, for every model $Y$ of $P_{2}$ and $Q_{2}$ ), $T_{P_{2}}(Y)=\{a\}=T_{Q_{2}}(Y)$. Thus, $P_{2}$ and $Q_{2}$ are suppequivalent relative to $\mathcal{H B}^{n}(A t, A t)$.

Finally, $Y \in \operatorname{Mod}_{A t \backslash\{b\}}\left(P_{3}\right)$ if and only if $Y \models P_{3}$ and $Y \backslash T_{P_{3}}(Y) \subseteq A t \backslash\{b\}$. Clearly, if $Y \models P_{3}$, then $T_{P_{3}}(Y)$ is defined and $b \notin Y$. Thus, $Y \backslash T_{P_{3}}(Y) \subseteq A t \backslash\{b\}$. It follows that $Y \in \operatorname{Mod}_{A t \backslash\{b\}}\left(P_{3}\right)$ if and only if $Y \models P_{3}$, that is, if and only if $a \in Y$ and $b \notin Y$. One can check that this condition also characterizes $Y \in \operatorname{Mod}_{A t \backslash\{b\}}\left(Q_{3}\right)$. Indeed, $Y \models Q_{3}$ if and only if $a \in Y$, and $Y \backslash T_{Q_{3}}(Y) \subseteq$ At $\backslash\{b\}$ if and only if $b \notin Y$. Thus, $\operatorname{Mod}_{A t \backslash\{b\}}\left(P_{3}\right)=$ $\operatorname{Mod}_{A t \backslash\{b\}}\left(Q_{3}\right)$. Moreover, if $Y \in \operatorname{Mod}_{A t \backslash\{b\}}\left(P_{3}\right)(a \in Y$ and $b \notin Y), T_{P_{3}}(Y)=\{a\}=T_{Q_{3}}(Y)$. Consequently, $P_{3}$ and $Q_{3}$ are supp-equivalent relative to $\mathcal{H B}^{n}(A t \backslash\{b\}, A t)$.

We conclude this section by generalizing our results to the disjunctive case.

Corollary 3.6 Let $P$ and $Q$ be disjunctive programs, and $A \subseteq A t$. Then, the following conditions are equivalent: 
1. $P$ and $Q$ are supp-equivalent relative to $\mathcal{H B}^{d}(A, A t)$

2. For every class $\mathcal{C}$ of context programs such that $\mathcal{H B}^{n}(A, \emptyset) \subseteq \mathcal{C} \subseteq \mathcal{H B}^{d}(A, A t), P$ and $Q$ are suppequivalent relative to $\mathcal{C}$

3. $\operatorname{sh}(P)$ and $\operatorname{sh}(Q)$ are supp-equivalent relative to $\mathcal{H B}^{n}(A, A t)$

4. $\operatorname{Mod}_{A}(\operatorname{sh}(P))=\operatorname{Mod}_{A}(\operatorname{sh}(Q))$ and for every $Y \in$ $\operatorname{Mod}_{A}(\operatorname{sh}(P)), T_{s h(P)}(Y)=T_{s h(Q)}(Y)$.

Proof. Since $\mathcal{C} \subseteq \mathcal{H} \mathcal{B}^{d}(A, A t)$, (1) implies (2). Thus, let us assume (2). Taking $\mathcal{C}=\mathcal{H B}^{n}(A, A t)$, we obtain that $P$ and $Q$ are supp-equivalent relative to $\mathcal{H B}^{n}(A, A t)$. Since shifting does not affect supported models, and since for every normal program $R, s h(R)=R$, we have (3). To show that (3) implies (1), let $R \in \mathcal{H} \mathcal{B}^{d}(A, A t)$ and let $M$ be a supported model of $P \cup R$. As supported models are not affected by shifting, $M$ is a supported model of $\operatorname{sh}(P \cup R)=$ $\operatorname{sh}(P) \cup \operatorname{sh}(R)$. Clearly, $\operatorname{sh}(R) \in \mathcal{H B}^{n}(A, A t)$. Thus, by (3), $M$ is a supported model of $\operatorname{sh}(Q) \cup \operatorname{sh}(R)$. Consequently, $M$ is a supported model of $Q \cup R$. By the symmetry argument, $P \cup R$ and $Q \cup R$ have the same supported models and that proves (1). It follows that (1), (2) and (3) are equivalent. To complete the proof, we note that the equivalence of (3) and (4) follows by Theorems 3.2.

Corollary 3.6 applies, in particular, to the cases when $\mathcal{C}$ is any of the following classes: $\mathcal{H B}^{d}(A, A t), \mathcal{H B}^{n}(A, A t)$, $\mathcal{H B}^{d}(A, \emptyset)$, and $\mathcal{H B}^{n}(A, \emptyset)$. It also implies an observation, already noted above, that the alphabet allowed for the bodies of context programs plays no role in the case of suppequivalence, unlike in the case of hyperequivalence with respect to stable models (Woltran 2008). In particular, for the semantics of supported models, there is no difference between strong and uniform equivalence (even for disjunctive programs).

Finally, we note that Theorem 3.2 also implies a characterization of uniform hyperequivalence with respect to stable models for tight logic programs (Fages 1994), as for such programs stable and supported models coincide (we refer to (Lee \& Lifschitz 2003) for a more detailed discussion of tight disjunctive logic programs and relevant results).

Corollary 3.7 Let $P$ and $Q$ be tight disjunctive programs, $A \subseteq A t$, and $\mathcal{C}$ a class of programs such that $\mathcal{H B}^{n}(A, \emptyset) \subseteq$ $\mathcal{C} \subseteq \mathcal{H} \mathcal{B}^{d}(A, \emptyset)$. Then, $P$ and $Q$ are uniformly equivalent (with respect to the stable-model semantics) relative to $\mathcal{C}$ if and only if $\operatorname{Mod}_{A}(\operatorname{sh}(P))=\operatorname{Mod}_{A}(\operatorname{sh}(Q))$ and for every $Y \in \operatorname{Mod}_{A}(\operatorname{sh}(P)), T_{s h(P)}(Y)=T_{s h(Q)}(Y)$.

Proof. Let $R \in \mathcal{H B}^{d}(A, \emptyset)$. Since $R$ consists of rules with the empty body, both $P \cup R$ and $Q \cup R$ are tight. Thus, they have the same stable models if and only if they have the same supported models. The assertion follows now from Corollary 3.6.

The characterization given by Corollary 3.7 provides an alternative to the characterization given in (Gebser et al. 2008).

\section{Hyperequivalence with respect to supported minimal models}

We move on to the semantics of supported minimal models. Let $\mathcal{C}$ be a class of programs. Disjunctive programs $P$ and $Q$ are suppmin-equivalent relative to a class $\mathcal{C}$ of disjunctive programs if for every program $R \in \mathcal{C}, P \cup R$ and $Q \cup R$ have the same suppmin models. As in the case of suppequivalence, we restrict attention to classes $\mathcal{C}$ that contain the empty program. This restriction ensures that suppminequivalence implies the equivalence with respect to suppmin models.

We note that suppmin-equivalence is a different concept than other types of equivalence we considered.

Example 4.1 The programs $P_{2}$ and $Q_{2}$ from Example 3.1 are suppmin-equivalent with respect to any class of programs, as for every program $R$, programs $P_{2} \cup R$ and $Q_{2} \cup R$ have the same models and the same supported models. However, as we pointed out earlier, they are not equivalent with respect to stable models nor hyperequivalent with respect to stable models relative to any class of programs.

Programs $P_{4}=P_{2}$ and $Q_{4}=\{a \leftarrow$ not $a\}$ have the same models, stable models, and are hyperequivalent with respect to stable models relative to an arbitrary class of programs. However, $P_{4}$ and $Q_{4}$ are not suppmin-equivalent (they have different suppmin models).

Next, one can show that for every set $U$ of atoms, programs $P_{1} \cup U$ and $Q_{1} \cup U$ have the same suppmin models, but the programs themselves have different supported models. Thus, $P_{1}$ and $Q_{1}$ are suppmin-equivalent relative to the class $\mathcal{H B}^{n}(A t, \emptyset)$ of all programs consisting of facts, but they are not supp-equivalent relative to the same class. We note that $P_{1}$ and $Q_{1}$ are not suppmin-equivalent relative to $\mathcal{H B}^{n}(A t, A t)$, as witnessed by the context $R=\{\leftarrow$ not a $\}$.

Finally, $P_{5}=\{a \leftarrow b ; b \leftarrow b ; \leftarrow \operatorname{not} a$, not $b\}$ and $Q_{5}=\{a \leftarrow b ; b \leftarrow a ; \leftarrow \operatorname{not} a$, not $b\}$ have the same supported models but different suppmin models $(\{a, b\}$ is the only supported model of $P_{5}$ and $Q_{5}$, and a suppmin model for $Q_{5}$ but not for $\left.P_{5}\right)$. Thus, the programs are suppequivalent relative to $\mathcal{H B}^{n}(\emptyset, \emptyset)$ (which contains the empty program only) but not suppmin-equivalent with respect to that class.

Our examples distinguishing between supp-and suppminequivalence refer to restricted classes of contexts. As we show later, it is not coincidental. The two types of equivalence are the same if all programs are allowed as contexts.

To characterize suppmin-equivalence relative to contexts in $\mathcal{H B}^{n}(A, B)$ and $\mathcal{H} \mathcal{B}^{d}(A, B)$, where $A, B \subseteq A t$, we use a refinement of the method from the previous section. As before, we focus first on the case of normal logic programs (and restrict the context to normal logic programs, as well). We recall that the characterization of supp-equivalence in that case is based on a relatively simple concept of an $A$ model of a program. For suppmin-equivalence, the second alphabet, $B$, has to be taken into consideration. Its role is reflected in the concepts of an $(A, B)$-model and an $e x$ tended $(A, B)$-model, that generalize the earlier notion of an $A$-model. 
We say that a set $Y$ of atoms is an $(A, B)$-model of a program $P$ if it satisfies the following two conditions:

1. $Y \in \operatorname{Mod}_{A}(P)$, that is, $Y$ is an $A$-model of $P$

2. for each $Z \subset Y$ such that $\left.Z\right|_{A \cup B}=\left.Y\right|_{A \cup B}, Z \not \models P$.

We say that a pair $(X, Y)$ of sets of atoms is an extended $(A, B)$-model of a normal program $P$ if $Y$ is an $(A, B)$ model of $P$ (satisfies conditions (1) and (2) above), and

3. $\left.X \subseteq Y\right|_{A \cup B}$

4. for each $Z \subset Y$ such that $\left.Z\right|_{B}=\left.X\right|_{B}$ and $\left.\left.Z\right|_{A} \supseteq X\right|_{A}$, $Z \not \models P$

5. if $\left.X\right|_{B}=\left.Y\right|_{B}$, then $Y \backslash T_{P}(Y) \subseteq X$.

We denote the set of all extended $(A, B)$-models of $P$ by $\operatorname{Mod}_{A}^{B}(P)$.

We start by discussing the intuitions behind $(A, B)$ models, extended $(A, B)$-models, and the conditions that define them. The role of the requirement that $Y$ be an $A$ model (the condition (1)) is the same as before. It captures the property that an interpretation can be turned into a supported model of an extension of a program with some program from $\mathcal{H B}^{n}(A, B)$. Thus, we focus here on the remaining conditions, which are meant to ensure that the requirement of minimality of models affects the programs under comparison in the same way.

First, we note that $(A, B)$-models of a program $P$ are precisely those interpretations that can be turned into a suppmin models of $P$ by extending it with a program from $\mathcal{H B}^{n}(A, B)$. More precisely, we have the following lemma.

Lemma 4.2 Let $P$ be a normal program, and $A, B \subseteq A t$. Then, there exists a program $R \in \mathcal{H} \mathcal{B}^{n}(A, B)$ such that $Y$ is a suppmin model of $P \cup R$ if and only if $Y$ is an $(A, B)$ model of $P$.

Proof. $(\Rightarrow)$ Let us assume that $Y$ is a suppmin model of $P \cup R$, where $R \in \mathcal{H B}^{n}(A, B)$. Then, $Y$ is a supported model of $P \cup R$. It follows that $Y$ is model of $P$ and $Y=$ $T_{P \cup R}(Y)=T_{P}(Y) \cup T_{R}(Y)$. Since $R \in \mathcal{H B}^{n}(A, B)$, $Y \backslash T_{P}(Y) \subseteq A$ and, consequently, $Y$ is an $A$-model of $P$ (we used this argument already in Section 3). Next, let us consider $Z \subset Y$ such that $\left.Z\right|_{A \cup B}=\left.Y\right|_{A \cup B}$. Since $Y \models R$ and $R \in \mathcal{H B}^{n}(A, B), Z \models R$. Since $Y$ is a minimal model of $P \cup R, Z \forall \forall P \cup R$. Thus, $Z \not \forall P$ follows and proves that $Y$ satisfies the condition (2) of the definition of an $(A, B)$ model of $P$.

$(\Leftarrow)$ For the "if" direction, let

$$
R=\left.\left.\left(Y \backslash T_{P}(Y)\right)\right|_{B} \cup Y\right|_{A \backslash B} \cup\left\{\leftarrow \operatorname{not} y|y \in Y|_{B}\right\} .
$$

It is easy to check that $Y \models R$. Next, since $Y$ is an $A$-model of $P, Y \models P$. Thus $Y \models P \cup R$. Moreover, we have $Y \backslash T_{P}(Y) \subseteq A$. It follows that

$T_{P}(Y) \cup T_{R}(Y)=\left.\left.T_{P}(Y) \cup\left(Y \backslash T_{P}(Y)\right)\right|_{B} \cup Y\right|_{A \backslash B}=Y$, which shows that $Y$ is a supported model of $P \cup R$. Now, let $Z \subseteq Y$ be such that $Z \models P \cup R$. Clearly, $\left.Y\right|_{A \backslash B} \subseteq Z$ ( $Z \models R$ and $R$ contains $\left.\left.Y\right|_{A \backslash B}\right)$. Moreover, the constraints in $R$ imply that $\left.\left.Y\right|_{B} \subseteq Z\right|_{B}$. As $Z \subseteq Y,\left.Y\right|_{A \cup B}=\left.Z\right|_{A \cup B}$. Since $Z \models P$ and $Y$ satisfies the condition (2) of the definition of an $(A, B)$-model of $P, Y=Z$. Hence, $Y$ is also a minimal model of $P \cup R$.
Lemma 4.2 and its proof imply immediately the following corollary providing a necessary condition for suppminequivalence of normal programs.

Corollary 4.3 Let $P$ and $Q$ be normal programs and $A, B \subseteq A$ t. If $P$ and $Q$ are suppmin-equivalent relative to $\mathcal{H B}^{n}(A, B)$, then $P$ and $Q$ have the same $(A, B)$-models. Moreover, for each $(A, B)$-model $Y$ of $P$ (and so, also of $Q),\left.T_{P}(Y)\right|_{B}=\left.T_{Q}(Y)\right|_{B}$.

Proof. The first part of the assertion follows directly from Lemma 4.2. To prove the second part of the assertion, let $Y$ be an $(A, B)$-model of $P$ and $R$ the program constructed in the proof of Lemma 4.2. We note that $T_{R}(Y)=(Y \backslash$ $\left.T_{P}(Y)\right)\left.\left.\right|_{B} \cup Y\right|_{A \backslash B}$.

By the proof of Lemma 4.2, $Y$ is a suppmin model of $P \cup$ $R$. Since $P$ and $Q$ are suppmin-equivalent, $Y$ is a suppmin model of $Q$, as well. It follows that $Y=T_{Q}(Y) \cup T_{R}(Y)$ and so,

$$
\begin{aligned}
\left.Y\right|_{B} & =\left.\left.T_{Q}(Y)\right|_{B} \cup T_{R}(Y)\right|_{B} \\
& =\left.\left.T_{Q}(Y)\right|_{B} \cup\left(Y \backslash T_{P}(Y)\right)\right|_{B} \\
& =\left.T_{Q}(Y)\right|_{B} \cup\left(\left.\left.Y\right|_{B} \backslash T_{P}(Y)\right|_{B}\right) .
\end{aligned}
$$

Since $Y \models P, T_{P}(Y) \subseteq Y$. Thus, $\left.\left.T_{P}(Y)\right|_{B} \subseteq T_{Q}(Y)\right|_{B}$. By the symmetry, $\left.T_{P}(Y)\right|_{B}=\left.T_{Q}(Y)\right|_{B}$.

The requirement stated in Corollary 4.3 is not sufficient. We still have to ensure that whenever adding a program $R \in \mathcal{H B}^{n}(A, B)$ to $P$ turns an $(A, B)$-model $Y$ of $P$ into a suppmin model of $P \cup R, Y$ (which, by our earlier discussion, must also be an $(A, B)$-model of $Q$ ) becomes a suppmin model of $Q \cup R$, as well (and vice versa). That is when extended $(A, B)$-models come into play. With each $(A, B)$ model $Y$ they associate some subsets $X$ of $Y$ that are needed to enforce the minimality of the model condition in extended programs. Due to the form of contexts, only those subsets of that are also subsets of $A \cup B$ are important. This restriction is reflected in the condition (3) of the definition of an extended $(A, B)$-model.

The essential part of the specification is provided by the conditions (4) and (5). They are designed so that each $(A, B)$-model $(X, Y)$ of a program $P$ would give rise to a particular context program $R$ with the following properties: $Y$ is a suppmin model of $P \cup R$; and for every program $Q$ either $(X, Y)$ is an extended $(A, B)$-model of $Q$ or $Y$ is $n o t$ a suppmin model of $Q$. The construction of $R$ depends on whether $\left.\left.X\right|_{B} \subset Y\right|_{B}$ or $\left.X\right|_{B}=\left.Y\right|_{B}$. There are no other possibilities as $X \subseteq Y$ follows from the condition (3) of the definition of an extended $(A, B)$-model.

Let $(X, Y)$ be an extended $(A, B)$-model of a normal logic program $P$, and let us assume that $\left.X\right|_{B} \subset Y_{B}$. We fix some $\left.\left.t \in Y\right|_{B} \backslash X\right|_{B}$ and define

$$
\begin{aligned}
R^{\subset}= & \left\{\left.y \leftarrow t\left|y \in\left(Y \backslash T_{P}(Y)\right)\right|_{B} \cup Y\right|_{A \backslash B}\right\} \cup \\
& \left\{x \leftarrow \text { not } t|x \in X|_{A}\right\} \cup\left\{\leftarrow \operatorname{not} x|x \in X|_{B}\right\} \cup \\
& \left\{\leftarrow u, \text { not }\left.z|u, z \in Y|_{B} \backslash X\right|_{B}\right\} .
\end{aligned}
$$

Clearly, $R^{\subset} \in \mathcal{H} \mathcal{B}(A, B)$. We will show that $R^{\subset}$ has the required properties. 
Lemma 4.4 Let $A, B$ be subsets of $A t, P, Q$ normal programs with the same $(A, B)$-models, and $(X, Y)$ an extended $(A, B)$-model of $P$ such that $\left.\left.X\right|_{B} \subset Y\right|_{B}$. Then, $Y$ is a suppmin model of $P \cup R \subset$, and $(X, Y)$ is an extended $(A, B)$-model of $Q$ or $Y$ is not a suppmin model of $Q \cup R \subset$.

Proof. Since $(X, Y)$ is an extended $(A, B)$-model of $P, Y$ is an $A$-model of $P$. Thus, $Y \models P$ and $T_{P}(Y) \subseteq Y$. Since $X \subseteq Y, Y \models R \subset$. Moreover, again by the fact that $Y$ is an $A$-model of $P, Y \backslash T_{P}(Y) \subseteq A$. Finally, since $t \in Y$, the rules $\left\{\left.y \leftarrow t\left|y \in\left(Y \backslash T_{P}(Y)\right)\right|_{B} \cup Y\right|_{A \backslash B}\right\}$ in $R \subset$ are applicable with respect to $Y$. It now follows that

$T_{P}(Y) \cup T_{R} \subset(Y)=\left.\left.T_{P}(Y) \cup\left(Y \backslash T_{P}(Y)\right)\right|_{B} \cup Y\right|_{A \backslash B}=Y$,

which shows that $Y$ is a supported model of $P \cup R^{\subset}$.

Now, let $Z \subseteq Y$ be such that $Z \models P \cup R^{\subset}$. It follows that $\left.\left.Z\right|_{B} \subseteq Y\right|_{B}$. Since $Z \models\left\{\leftarrow\right.$ not $\left.x|x \in X|_{B}\right\},\left.X\right|_{B} \subseteq$ $\left.Z\right|_{B}$. Thus, since $Z \models\left\{\leftarrow u\right.$, not $\left.\left.z|u, z \in Y|_{B} \backslash X\right|_{B}\right\}$, we obtain that $\left.Z\right|_{B}=\left.Y\right|_{B}$ or $\left.Z\right|_{B}=\left.X\right|_{B}$.

First, we consider the case $\left.Z\right|_{B}=\left.Y\right|_{B}$. Since $Z \models$ $R \subset$, we have $\left.Y\right|_{A \backslash B} \subseteq Z$ (thanks to the rules $y \leftarrow t$, $\left.y \in Y\right|_{A \backslash B}$, in $R^{\complement}$ ). It follows that $\left.Y\right|_{A \cup B} \subseteq Z$. Consequently, $\left.\left.Y\right|_{A \cup B} \subseteq Z\right|_{A \cup B}$. On the other hand $Z \subseteq Y$ and so, $\left.\left.Z\right|_{A \cup B} \subseteq \bar{Y}\right|_{A \cup B}$. Thus, $\left.Y\right|_{A \cup B}=\left.Z\right|_{A \cup B}$. Since $Z \models P$ and $Y$ satisfies the condition (2) of the definition of an $(A, B)$-model of $P, Y=Z$.

Next, we consider the case $\left.Z\right|_{B}=\left.X\right|_{B}$. We have $\left.X\right|_{A} \subseteq$ $Z$ (thanks to the rules $x \leftarrow \operatorname{not} t,\left.x \in X\right|_{A}$, in $R^{\subset}$ ). Since $Z \models P$ and $(X, Y)$ is an extended $(A, B)$-model of $P$, the condition (4) implies $Y=Z$. Thus, in each case, $Y=Z$ and, consequently, $Y$ is a minimal model of $P \cup R^{\subset}$. It follows that $Y$ is a suppmin model of $P \cup R^{\complement}$.

To show the second part of the assertion, let us assume that $(X, Y)$ is not an extended $(X, Y)$-model of $Q$. Since $P$ and $Q$ have the same $(A, B)$-models, $(X, Y)$ violates the condition (4) of the definition of an $(X, Y)$-model. That is, there is $Z \subset Y$ such that $\left.X\right|_{B}=\left.Z\right|_{B},\left.\left.Z\right|_{A} \supseteq X\right|_{A}$ and $Z \models Q$.

We observe that $Z \models R \subset$. Indeed, $t \notin Z$ (we have $t \in B$, $\left.Z\right|_{B}=\left.X\right|_{B}$ and $\left.t \notin X\right|_{B}$ ). Thus, $Z$ is a model of all rules in $R$ with a positive occurrence of $t$ in the body. Since $\left.X\right|_{A} \subseteq$ $\left.Z\right|_{A}$, the rules $x \leftarrow$ not $t$ are satisfied by $Z$, too. Finally, as $\left.Z\right|_{B}=\left.X\right|_{B}$, the constraints in $\left\{\leftarrow\right.$ not $\left.x|x \in X|_{B}\right\} \cup\{\leftarrow$ $u$, not $\left.\left.z|u, z \in Y|_{B} \backslash X\right|_{B}\right\}$ in $R^{\subset}$ are satisfied, as well. It follows that $Z \models Q \cup R^{\subset}$. Consequently, $Y$ is not a minimal model of $Q \cup R \subset$.

The next lemma addresses the case $\left.X\right|_{B}=\left.Y\right|_{B}$. Here, the condition (5) comes into play and we need to use a different context. Let $(X, Y)$ be an extended $(A, B)$-model of a normal logic program $P$, and let us assume that $\left.X\right|_{B}=Y_{B}$. We define

$$
\begin{aligned}
R^{=}= & \left.\left.\left(Y \backslash T_{P}(Y)\right)\right|_{B} \cup X\right|_{A \backslash B} \cup \\
& \left\{\leftarrow \text { not } y|y \in Y|_{B}\right\} .
\end{aligned}
$$

Again it is evident that $R^{=} \in \mathcal{H B}^{n}(A, B)$.

Lemma 4.5 Let $A, B$ be subsets of $A t, P, Q$ normal programs with the same $(A, B)$-models, and $(X, Y)$ an extended $(A, B)$-model of $P$ such that $\left.X\right|_{B}=\left.Y\right|_{B}$. Then,
$Y$ is a suppmin model of $P \cup R^{=}$, and $(X, Y)$ is an extended $(A, B)$-model of $Q$ or $Y$ is not a suppmin model of $Q \cup R^{=}$.

Proof. Since $(X, Y)$ is an extended $(A, B)$-model of $P, Y$ is an $A$-model of $P$, and hence $Y \models P$. One can check that $Y \models R^{=}$, as well. Since $Y \models P \cup R^{=}, T_{P \cup R}=(Y) \subseteq Y$. To show that $Y$ is a supported model of $P \cup R^{=}$, we now prove $Y \subseteq T_{P \cup R^{\prime}}(Y)$. Let $y \in Y \backslash T_{P}(Y)$. It follows that $y \in X$ (by the condition (5) of the definition of an extended $(A, B)$ model for $P$ ) and $y \in A$ (by the fact that $Y$ is an $A$-model of $P$ ). If $y \notin B$, then $\left.y \in X\right|_{A \backslash B}$ and so, $y \in T_{R}=(Y)$. If $y \in B$, then $\left.y \in\left(Y \backslash T_{P}(Y)\right)\right|_{B}$. Thus, $y \in T_{R}=(Y)$ in this case, too. It follows that if $y \in Y$ then $y \in T_{P}(Y) \cup T_{R}=(Y)$ and consequently, $Y \subseteq T_{P}(Y) \cup T_{R}=(Y)=T_{P \cup R}=(Y)$.

We will now show that $Y$ is a minimal model of $P \cup R^{=}$. To this end, let us consider $Z \subseteq Y$ such that $Z \models P \cup R^{=}$. It follows that $\left.Z\right|_{B}=\left.Y\right|_{B}$ (since $Z$ is a model of the constraints in $R^{=}$). Moreover, $\left.X\right|_{A \backslash B} \subseteq Z$. If $\left.y \in X\right|_{A \cap B}$, then $\left.y \in X\right|_{B}$ and $\left.y \in Y\right|_{B}$. Thus, $\left.y \in Z\right|_{B}$. It follows that $\left.X\right|_{A} \subseteq Z$ and, consequently, $\left.\left.X\right|_{A} \subseteq Z\right|_{A}$. Moreover, $\left.X\right|_{B}=\left.Y\right|_{B}$ implies that $\left.Z\right|_{B}=\left.X\right|_{B}$. Since $Z \models P$ and $(X, Y)$ is an extended $(A, B)$-model of $P$ (satisfies, in particular, the condition (4)), $Z=Y$. Thus, $Y$ is a minimal model of $P \cup R^{=}$.

To prove the second part of the assertion, let us assume that $Y$ is a suppmin model of $Q \cup R^{=}$. We will show that $(X, Y)$ is an extended $(A, B)$-model of $Q$. Since $P$ and $Q$ have the same $(A, B)$-models and $\left.X \subseteq Y\right|_{A \cup B}$ (by the fact that $(X, Y)$ is an extended $(A, B)$-model of $P)$, it suffices to show that the conditions (4) and (5) hold.

For the condition (5), we proceed as follows. By the assumption, $Y$ is a supported model of $Q \cup R^{=}$. Thus, $Y=T_{Q \cup R}=(Y)=T_{Q}(Y) \cup T_{R}=(Y)$. Let $y \in Y \backslash T_{Q}(Y)$. We have $y \in T_{R}=(Y)$. Consequently, $y \in Y \backslash T_{P}(Y)$ or $y \in X$. Since $(X, Y)$ is an extended $(A, B)$-model of $P$ and $\left.X\right|_{B}=\left.Y\right|_{B}, Y \backslash T_{P}(Y) \subseteq X$. Thus, $y \in X$ and so $Y \backslash T_{Q}(Y) \subseteq X$.

For the condition (4), we consider a set $Z \subset Y$ such that $\left.Z\right|_{B}=\left.X\right|_{B}$ and $\left.\left.Z\right|_{A} \supseteq X\right|_{A}$. Since $\left.Y\right|_{B}=\left.X\right|_{B},\left.Z\right|_{B}=$ $\left.Y\right|_{B}$. Thus, $Z \models R^{=}$. Since $Y$ is a minimal model of $Q \cup R$, $Z \not \forall Q$ follows.

Our discussion provided some insights into the conditions defining the notion of an extended $(A, B)$-model and the role they play. Along the way we also derived several necessary conditions for $P$ and $Q$ to be suppmin-equivalent. The following theorem, our main result of this section, shows that these conditions together are also sufficient.

Theorem 4.6 Let $A, B \subseteq$ At and let $P, Q$ be normal programs. The following conditions are equivalent

1. $P$ and $Q$ are suppmin-equivalent relative to $\mathcal{H B}^{n}(A, B)$

2. $\operatorname{Mod}_{A}^{B}(P)=\operatorname{Mod}_{A}^{B}(Q)$ and for every $(X, Y) \in$ $\operatorname{Mod}_{A}^{B}(P),\left.T_{P}(Y)\right|_{B}=\left.T_{Q}(Y)\right|_{B}$

3. $\operatorname{Mod}_{A}^{B}(P)=\operatorname{Mod}_{A}^{B}(Q)$ and for every $(X, Y) \in$ $\operatorname{Mod}_{A}^{B}(P), T_{P}(Y) \backslash(A \backslash B)=T_{Q}(Y) \backslash(A \backslash B)$.

Proof. We start by showing that (2) and (3) are equivalent. Indeed, in either case, if $(X, Y) \in \operatorname{Mod}_{A}^{B}(P)$, 
then $T_{P}(Y) \subseteq Y, Y \backslash T_{P}(Y) \subseteq A, T_{Q}(Y) \subseteq Y$ and $Y \backslash T_{Q}(Y) \subseteq A$. Consequently, we have

$$
T_{P}(Y) \backslash(A \backslash B)=\left.T_{P}(Y)\right|_{B} \cup(Y \backslash(A \cup B))
$$

and

$$
T_{Q}(Y) \backslash(A \backslash B)=\left.T_{Q}(Y)\right|_{B} \cup(Y \backslash(A \cup B)) .
$$

Since in either case the sets involved in the union are disjoint, $\left.T_{P}(Y)\right|_{B}=\left.T_{Q}(Y)\right|_{B}$ if and only if $T_{P}(Y) \backslash(A \backslash B)=$ $T_{Q}(Y) \backslash(A \backslash B)$. Thus, the equivalence of (2) and (3) follows. We complete the proof by showing the equivalence of (1) and (2).

[(1) implies (2)]: By Corollary 4.3, $P$ and $Q$ have the same $(A, B)$-models. Let $(X, Y) \in \operatorname{Mod}_{A}^{B}(P)$ be an extended $(A, B)$-model of $P$. Since $P$ and $Q$ are suppmin-equivalent, Lemmas 4.4 and 4.5 imply that $(X, Y) \in \operatorname{Mod}_{A}^{B}(Q)$. By the symmetry, $\operatorname{Mod}_{A}^{B}(P)=\operatorname{Mod}_{A}^{B}(Q)$ follows. Moreover, if $(X, Y) \in \operatorname{Mod}_{A}^{B}(P)$ then $Y$ is an $(A, B)$-model of $P$ and so, by Corollary 4.3, $\left.T_{P}(Y)\right|_{B}=\left.T_{Q}(Y)\right|_{B}$.

[(2) implies (1)]: Let $R$ be a logic program from $\mathcal{H B}^{n}(A, B)$, and let $Y$ be a supported minimal model of $P \cup R$. Next, let $X=\left.T_{R}(Y) \cup Y\right|_{B}$. We note that since $Y \models R, T_{R}(Y) \subseteq Y$. Thus, $\left.X\right|_{B}=\left.Y\right|_{B}$.

We will show that $(X, Y) \in \operatorname{Mod}_{A}^{B}(P)$. Since $Y$ is a suppmin model of $P \cup R$, it follows that $Y \models P, Y \models R$, and $Y=T_{P}(Y) \cup T_{R}(Y)$. We have $R \in \mathcal{H} \mathcal{B}^{n}(A, B)$. Thus, the latter identity shows that $Y \backslash T_{P}(Y) \subseteq A$. Since $Y \models P$, we obtain that $Y \in \operatorname{Mod}_{A}(P)$, that is, the condition (1) for $(X, Y) \in \operatorname{Mod}_{A}^{B}(P)$ holds.

Since $T_{R}(Y) \subseteq Y$ and $T_{R}(Y) \subseteq A$ (we recall that $R \in$ $\left.\mathcal{H B}^{n}(A, B)\right),\left.\left.T_{R}(Y) \subseteq Y\right|_{A} \subseteq Y\right|_{A \cup B}$. Clearly, $\left.Y\right|_{B} \subseteq$ $\left.Y\right|_{A \cup B}$. Thus, $\left.X \subseteq Y\right|_{A \cup B}$. This proves that the condition (3) for $(X, Y) \in \operatorname{Mod}_{A}^{B}(P)$ holds.

We also have $T_{R}(Y) \subseteq X$ (by the definition of $X$ ). Since $Y \backslash T_{P}(Y) \subseteq T_{R}(Y), \bar{Y} \backslash T_{P}(Y) \subseteq X$ follows. Consequently, the condition (5) for $(X, Y) \in \operatorname{Mod}_{A}^{B}(P)$ holds, too.

Next, let $Z \subset Y$ and $\left.Z\right|_{A \cup B}=\left.Y\right|_{A \cup B}$. Since $R \in$ $\mathcal{H B}^{n}(A, B)$ and $Y \models R, Z \models R$. We have that $Y$ is a minimal model of $P \cup R$. Thus, $Z \not \models P$ and, consequently, the condition (2) for $(X, Y) \in \operatorname{Mod}_{A}^{B}(P)$ follows.

Finally, let $Z \subset Y,\left.Z\right|_{B}=\left.X\right|_{B}$ and $\left.\left.Z\right|_{A} \supseteq X\right|_{A}$. Since $\left.X\right|_{B}=\left.Y\right|_{B},\left.Z\right|_{B}=\left.Y\right|_{B}$. We have $R \in \mathcal{H B}^{n}(A, B)$. Thus, $T_{R}(Z)=T_{R}(Y) \subseteq X$ (the inclusion holds by the definition of $X)$. Moreover, $T_{R}(Y) \subseteq A$ and so, $T_{R}(Z) \subseteq$ $\left.\left.X\right|_{A} \subseteq Z\right|_{A} \subseteq Z$. Consequently, $Z \models R$ in this case, too. As before, we obtain that $Z \not \models P$. This shows the condition (4) for $(X, Y) \in \operatorname{Mod}_{A}^{B}(P)$.

Thus, we have established that $(X, Y) \in \operatorname{Mod}_{A}^{B}(P)$. By the assumption, $(X, Y) \in \operatorname{Mod}_{A}^{B}(Q)$ and also $\left.T_{P}(Y)\right|_{B}=$ $\left.T_{Q}(Y)\right|_{B}$. We will now show that $Y=T_{Q}(Y) \cup T_{R}(Y)$, that is, that $Y$ is a supported model of $Q \cup R$. Since $Y \in$ $\operatorname{Mod}_{A}(Q), Y \models Q$. Hence, $Y \models Q \cup R$ (we recall that $Y \models R$ ) and so, $T_{Q}(Y) \cup T_{R}(Y) \subseteq Y$.

To show $Y \subseteq T_{Q}(Y) \cup T_{R}(Y)$, let $y \in Y$. We recall that $T_{R}(Y) \subseteq A$. We distinguish three cases: (i) $y \notin A$ : Since $(X, Y) \in \operatorname{Mod}_{A}^{B}(Q), Y \in \operatorname{Mod}_{A}(Q)$ and so, $Y \backslash T_{Q}(Y) \subseteq A$. Thus, $y \in T_{Q}(Y)$ follows.

(ii) $y \in B$ : If $y \in T_{R}(Y)$ we are done; otherwise (since $Y=$ $T_{P}(Y) \cup T_{R}(Y)$ ), we obtain $y \in T_{P}(Y)$. It follows that $\left.y \in T_{P}(Y)\right|_{B}$. Thus, $\left.y \in T_{Q}(Y)\right|_{B}$ and, consequently, $y \in T_{Q}(Y)$.

(iii) $y \in A \backslash B$ : If $y \in X$, then $y \in T_{R}(Y)$ (we recall that $\left.X=\left.T_{R}(Y) \cup Y\right|_{B}\right)$; if $y \notin X$, then $y \notin Y \backslash T_{Q}(Y)$ (indeed, since $(X, Y) \in \operatorname{Mod}_{A}^{B}(Q)$ and $\left.X\right|_{B}=\left.Y\right|_{B}$, $\left.Y \backslash T_{Q}(Y) \subseteq X\right)$, and thus, $y \in T_{Q}(Y)$.

It follows that $Y=T_{Q}(Y) \cup T_{R}(Y)$, that is, $Y$ is a supported model of $Q \cup R$.

It remains to show that $Y$ is a minimal model of $Q \cup R$. Let $Y^{\prime} \subset Y$ be a model of $Q \cup R$. Since $Y^{\prime} \models Q,\left(\left.Y^{\prime}\right|_{A \cup B}, Y\right) \notin \operatorname{Mod}_{A}^{B}(Q)$ (it violates condition (4) of the definition of $\left.\operatorname{Mod}_{A}^{B}(Q)\right)$. By the assumption, $\left(\left.Y^{\prime}\right|_{A \cup B}, Y\right) \notin \operatorname{Mod}_{A}^{B}(P)$. Since $(X, Y) \in \operatorname{Mod}_{A}^{B}(P)$, $\left(\left.Y^{\prime}\right|_{A \cup B}, Y\right)$ satisfies condition (1) of the definition of $\operatorname{Mod}_{A}^{B}(P)$. Moreover, $Y^{\prime} \subset Y$ implies $\left.\left.Y^{\prime}\right|_{A \cup B} \subseteq Y\right|_{A \cup B}$. Thus, condition (3) holds, too.

Let $\left.Y\right|_{B}=\left.\left(\left.Y^{\prime}\right|_{A \cup B}\right)\right|_{B}$. Then, $\left.Y\right|_{B}=\left.Y^{\prime}\right|_{B}$. Since $Y^{\prime} \models R$ and $R \in \mathcal{H B}^{n}(A, B), T_{R}\left(Y^{\prime}\right) \subseteq Y^{\prime}$ and $T_{R}\left(Y^{\prime}\right)=$ $T_{R}(Y)$. Thus, $T_{R}(Y) \subseteq Y^{\prime}$ and, consequently, $X \subseteq Y^{\prime}$. We proved above that $Y \backslash T_{P}(Y) \subseteq X$. Consequently, condition (5) for $\left(\left.Y^{\prime}\right|_{A \cup B}, Y\right) \in \operatorname{Mod}_{A}^{B}(P)$ holds, as well. It follows that at least one of the conditions (2) and (4) is violated. That is, there is $U \subset Y$, such that $U \models P$, and either $\left.U\right|_{A \cup B}=\left.Y\right|_{A \cup B}$ or $\left.U\right|_{B}=\left.Y^{\prime}\right|_{B}$ and $\left.\left.U\right|_{A} \supseteq Y^{\prime}\right|_{A}$. Since both $Y \models R$ and $Y^{\prime} \models R, U \models R$ (we recall here that $R \in \mathcal{H} \mathcal{B}^{n}(A, B)$ ). Thus, $U \models P \cup R$ and $Y$ is not a minimal model of $P \cup R$, a contradiction. It follows that there is no $Y^{\prime} \subset Y$ such that $Y^{\prime} \models Q \cup R$. That is, $Y$ is a supported minimal model of $Q \cup R$.

We have several corollaries for some special choices of $A$ and $B$. The first one concerns the case when $B=\emptyset$, that is, the case of relativized uniform suppmin-equivalence. Since the condition $\left.T_{P}(Y)\right|_{B}=\left.T_{Q}(Y)\right|_{B}$ is now trivially satisfied, Theorem 4.6 implies the following result.

Corollary 4.7 Let $A \subseteq$ At. Normal programs $P$ and $Q$ are suppmin-equivalent relative to $\mathcal{H B}^{n}(A, \emptyset)$ if and only if $\operatorname{Mod}_{A}^{\emptyset}(P)=\operatorname{Mod}_{A}^{\emptyset}(Q)$.

Proof. The result follows by the equivalence of the conditions (1) and (2) in Theorem 4.6.

Moreover, the description of $\operatorname{Mod}_{A}^{B}(P)$, when $B=\emptyset$ simplifies. In fact, $(X, Y) \in \operatorname{Mod}_{A}^{\emptyset}(P)$ if and only if

1. $Y \in \operatorname{Mod}_{A}(P)$

2. $\left.X \subseteq Y\right|_{A}$

3. for each $Z$ with $X \subseteq Z \subset Y, Z \not \forall P$

4. $Y \backslash T_{P}(Y) \subseteq X$.

When $A=B=A t$ (strong suppmin-equivalence), it turns out that supp-equivalence and suppmin-equivalence coincide (cf. comments at the end of Example 4.1). 
Corollary 4.8 Normal programs $P$ and $Q$ are suppminequivalent relative to $\mathcal{H B}^{n}(A t, A t)$ if and only if $P$ and $Q$ are supp-equivalent relative to $\mathcal{H B}^{n}(A t, A t)$.

Proof. We note that $(X, Y) \in \operatorname{Mod}_{A t}^{A t}(P)$ if and only if $Y \in \operatorname{Mod}_{A t}(P)$, and either $X=Y$, or $X \subset Y$ and $X \not \models P$. Thus, $\operatorname{Mod}_{A t}^{A t}(P)=\operatorname{Mod}_{A t}^{A t}(Q)$ if and only if $\operatorname{Mod}_{A t}(P)=$ $\operatorname{Mod}_{A t}(Q)$. Moreover, $(Y, Y) \in \operatorname{Mod}_{A t}^{A t}(P)$ if and only if $Y \in \operatorname{Mod}_{A t}(P)$. Thus, the result follows from Corollary 3.3 and Theorem 4.6.

We will discuss additional special-cases for instantiating $\operatorname{Mod}_{A}^{B}(P)$ as part of our complexity analysis in Lemma 6.1.

We will now use our results to resolve the issue of suppmin-equivalence of programs discussed earlier.

Example 4.9 If $P$ is a program such that every set of atoms is a model of $P$, then $\operatorname{Mod}_{A t}^{\emptyset}(P)=\{(Y, Y) \mid Y \subseteq A t\}$. This observation applies both to $P_{1}$ and $Q_{1}$. Thus, by Corollary 4.7, $P_{1}$ and $Q_{1}$ are suppmin-equivalent relative to $\mathcal{H B}^{n}(A t, \emptyset)$. We note that $P_{1}$ and $Q_{1}$ are not suppminequivalent relative to $\mathcal{H B}^{n}(A t, A t)$. Indeed, they are not supp-equivalent (cf. Example 3.5) and so, not suppminequivalent (by Corollary 4.8).

Next, we consider programs $P_{5}$ and $Q_{5}$. We note that for every program $P, \operatorname{Mod}_{\emptyset}^{\emptyset}(P)$ consists of pairs $(\emptyset, Y)$, where $Y$ is a suppmin model of $P$. Thus, $\operatorname{Mod}_{\emptyset}^{\emptyset}\left(P_{5}\right)=\emptyset$ and $\operatorname{Mod}_{\emptyset}^{\emptyset}\left(Q_{5}\right)=\{(\emptyset,\{a, b\})\}$. By Corollary 4.7, $P_{5}$ and $Q_{5}$ are not suppmin-equivalent relative to $\mathcal{H B}^{n}(\emptyset, \emptyset)$.

We will now consider the general case of disjunctive programs $P$ and $Q$, and the class of contexts $\mathcal{H} \mathcal{B}^{d}(A, B)$. We will first show that when considering suppmin-equivalence with respect to $\mathcal{H} \mathcal{B}^{d}(A, B)$, we can restrict to contexts in $\mathcal{H B}^{n}(A, B)$.

Lemma 4.10 Let $A, B \subseteq$ At and let $P$ and $Q$ be disjunctive programs. Then, $P$ and $Q$ are suppmin-equivalent with respect to $\mathcal{H B}^{d}(A, B)$ if and only if $P$ and $Q$ are suppminequivalent with respect to $\mathcal{H B}^{n}(A, B)$.

Proof. The "only-if" part of the assertion is evident. We will prove the "if" part only. Thus, let us assume that $P$ and $Q$ are suppmin-equivalent with respect to $\mathcal{H B}^{n}(A, B)$. Let $R \in \mathcal{H B}^{d}(A, B)$ and let $Y$ be a suppmin model of $P \cup R$. We will show that $Y$ is a suppmin model of $Q \cup R$.

A normal rule $r^{\prime}$ is a $Y$-split of a rule $r$ if (i) $b d\left(r^{\prime}\right)=$ $b d(r)$, and (ii) $h d\left(r^{\prime}\right)=\perp$ if $h d(r) \cap Y=\emptyset$, and $h d\left(r^{\prime}\right) \in$ $Y$, otherwise. A normal program $R^{\prime}$ if a $Y$-split of $R$ if $R^{\prime}$ is obtained from $R$ by replacing each rule in $R$ by one of its $Y$-splits.

Observation 1: For every $Y$-split $R^{\prime}$ of $R, Y$ is a model of $R^{\prime}$, and for every $Z \subseteq A t$, if $Z \models R^{\prime}$, then $Z \models R$.

Observation 2: For every $Y$-split $R^{\prime}$ of $R, Y$ is a suppmin model of $P \cup R^{\prime}$.

To prove that, we argue as follows. First, $Y \models P$ (as $Y$ is a suppmin model of $P \cup R$ ) and so, by Observation 1, $Y \models P \cup R^{\prime}$. Let $y \in Y$. Since $Y$ is a supported model of $P \cup R$, there is a rule $r \in P \cup R$ such that $Y \models b d(r)$ and $\{y\}=h d(r) \cap Y$. If $r \in R, y \leftarrow b d(r)$ belongs to $R^{\prime}$ (as it is the only $Y$-split of $r$ ). It follows that there is a rule $r^{\prime} \in P \cup R^{\prime}$ such that $Y \models b d\left(r^{\prime}\right)$ and $\{y\}=h d\left(r^{\prime}\right) \cap Y$ ( $r$, if $r \in P$; the split of $r, y \leftarrow b d(r)$, otherwise). Thus, $Y$ is a supported model of $P \cup R^{\prime}$. If $Z \subseteq Y$ and $Z \models P \cup R^{\prime}$, then, by Observation $1, Z \models P \cup R$. Since $Y$ is a suppmin model of $P \cup R, Z=Y$. Thus, $Y$ is a suppmin model of $P \cup R^{\prime}$, as claimed.

Since $Y$-splits are normal programs in $\mathcal{H B}^{n}(A, B)$, and since $P$ and $Q$ are suppmin-equivalent with respect to $\mathcal{H B}^{n}(A, B)$, it follows that for every $Y$-split $R^{\prime}$ of $R, Y$ is a suppmin model of $Q \cup R^{\prime}$. We will show that $Y$ is a supported model of $Q \cup R$.

Since the set of $Y$-splits of $R$ is nonempty, it follows from the statement above that $Y$ is a model of $Q$. Thus, $Y \models$ $Q \cup R$ (we recall that $Y$ is a suppmin model of $P \cup R$ and so, $Y$ is a model of $R$ ). We will now show that every $y \in Y$ there is a rule $r \in Q \cup R$ such that $Y \models b d(r)$ and $\{y\}=$ $h d(r) \cap Y$. To this end, let us assume that $R$ contains no such rule. Since $Y \models R$, for every $r \in R$ such that $Y \models b d(r)$, there is $y_{r} \in Y$ such that $y \neq y_{r}$ and $y_{r} \in h d(r)$. Let us consider the split $R^{\prime}$ of $R$ that replaces each such rule $r$ with the rule $y_{r} \leftarrow b d(r)$. It follows that $y \notin h d(R)$. Since $Y$ is a supported model of $Q \cup R^{\prime}$, there is a rule $r \in Q$ such that $Y \models b d(r)$ and $\{y\}=h d(r) \cap Y$. Thus, $Y$ is a supported model of $Q \cup R$.

Finally, we will show that $Y$ is a minimal model of $Q \cup R$. Let $Z \subseteq Y$ be a model of $Q \cup R$. For every rule $r \in R$ such that $Z \models b d(r)$, let $y_{r}$ be an element in the head of $r$ such that $y_{r} \in Z$. Let $R^{\prime}$ be any $Y$-split of $R$ which, for every such rule $r$ uses its $Y$-split $y_{r} \leftarrow b d(r)$. Then, $Z \models Q \cup R^{\prime}$. Since $Y$ is a suppmin model of $Q \cup R^{\prime}, Z=Y$. Thus, $Y$ is a minimal model of $Q \cup R$.

It follows that $Y$ is a suppmin model of $Q \cup R$. By the symmetry argument, $P \cup R$ and $Q \cup R$ have the same suppmin models. That is, $P$ and $Q$ are suppmin-equivalent with respect to $\mathcal{H B}^{d}(A, B)$.

We recall that shifting does not affect models and supported models of a program . Moreover, for every program $R \in \mathcal{H B}^{n}(A, B), \operatorname{sh}(R)=R$. Thus, we have the following result.

Corollary 4.11 Let $A, B \subseteq$ At and let $P$ and $Q$ be disjunctive programs. Then, $P$ and $Q$ are suppmin-equivalent with respect to $\mathcal{H B}^{d}(A, B)$ if and only if $\operatorname{sh}(P)$ and $\operatorname{sh}(Q)$ are suppmin-equivalent with respect to $\mathcal{H B}^{n}(A, B)$.

Thanks to Corollary 4.11, all results concerning suppminequivalence of normal programs with respect to normal contexts lift to the disjunctive case. We present two such results below.

Corollary 4.12 Let $A, B \subseteq A t$. The following conditions are equivalent.

1. Disjunctive programs $P$ and $Q$ are suppmin-equivalent relative to $\mathcal{H B}^{d}(A, B)$

2. $\operatorname{Mod}_{A}^{B}(\operatorname{sh}(P))=\operatorname{Mod}_{A}^{B}(\operatorname{sh}(Q))$ and for every $(X, Y) \in$ $\operatorname{Mod}_{A}^{B}(\operatorname{sh}(P)),\left.T_{\operatorname{sh}(P)}(Y)\right|_{B}=\left.T_{s h(Q)}(Y)\right|_{B}$. 
3. $\operatorname{Mod}_{A}^{B}(\operatorname{sh}(P))=\operatorname{Mod}_{A}^{B}(\operatorname{sh}(Q))$ and for every $(X, Y) \in$ $\operatorname{Mod}_{A}^{B}(\operatorname{sh}(P)), T_{s h(P)}(Y) \backslash(A \backslash B)=T_{s h(Q)}(Y) \backslash(A \backslash$ $B)$

Proof. This result follows by Corollary 4.11 from Theorem 4.6.

Corollary 4.13 Disjunctive programs $P$ and $Q$ are suppmin-equivalent relative to $\mathcal{H B}^{d}(A t, A t)$ if and only if $P$ and $Q$ are supp-equivalent relative to $\mathcal{H B}^{d}(A t, A t)$.

Proof. This result follows by Corollaries 4.11, 3.6, and 4.8.

\section{Complexity of Supp-Equivalence}

We focus entirely on the case of normal programs and normal contexts. As we noted, it is not an essential restriction, and all results we obtain hold without it. We will study deciding hyperequivalence relative to classes $\mathcal{H B}^{n}(A, B)$. Specifically, we will consider the following problems:

1. SUPP: given programs $P, Q$ (over $A t$ ) and $A, B \subseteq A t$, decide whether $P$ and $Q$ are supp-equivalent relative to $\mathcal{H B}^{n}(A, B)$

2. $\operatorname{SUPP}_{A}$ : given programs $P, Q$ (over $A t$ ) and $B \subseteq A t$, decide whether $P$ and $Q$ are supp-equivalent relative to $\mathcal{H B}^{n}(A, B)$

3. SUPP $^{B}$ : given programs $P, Q$ (over $A t$ ) and $A \subseteq A t$, decide whether $P$ and $Q$ are supp-equivalent relative to $\mathcal{H B}^{n}(A, B)$

4. $\operatorname{SUPP}_{A}^{B}$ : given programs $P, Q$ (over $A t$ ), decide whether $P$ and $Q$ are supp-equivalent relative to $\mathcal{H B}^{n}(A, B)$.

We emphasize the changing roles of the sets $A$ and $B$. In some cases, they are used to specify a problem ( $A$ in $\operatorname{SUPP}_{A}^{B}$ and $\left.\operatorname{SUPP}_{A}\right)$; in others, they belong to the specification of an instance ( $A$ in $\operatorname{SUPP}^{B}$ and SUPP). In the first role, they can be finite or infinite. For instance, SUPP $A t$ denotes the problem to decide, given programs $P, Q$ (over $A t$ ) and $B \subseteq A t$, whether $P$ and $Q$ are supp-equivalent relative to $\mathcal{H} \mathcal{B}^{n}(A t, B)$. In the second role, they need to have finite representations.

To establish the complexity of a problem, we derive an upper and a lower bound (membership and hardness). We start by pointing out that establishing an upper bound is not entirely straightforward. A natural witness against suppequivalence (relative to a class $\mathcal{C}$ ) is a pair $(R, Y)$, where $R$ is a finite program in $\mathcal{C}$ and $Y$ is finite set of atoms such that $Y$ is a supported model of exactly one of $P \cup R$ and $Q \cup R$. The problem is that the size of such a program $R$ might not be bounded by a polynomial in the size of $P, Q$, and possibly also $A$ and $B$, depending on the problem. Thus, the most direct attempt to prove the membership of the problem in the class coNP fails. The bound can, however, be derived from our characterization theorem for several classes of context programs.

Theorem 5.1 The following problems are in the class coNP:

1. SUPP
2. $\operatorname{SuPP}_{A}$, for every finite $A \subseteq A t$

3. $\operatorname{SUPP}_{A}^{B}$, for every finite $A \subseteq A$, and for every $B \subseteq A t$

4. $\mathrm{SUPP}^{B}$, for every $B \subseteq A t$

5. $\operatorname{SUPP}_{A t}^{B}$, for every $B \subseteq A t$.

Proof. (1) Theorem 3.2 implies that the complement of the problem is in the class NP. Indeed, given two programs $P$ and $Q$ and sets $A, B \subseteq A t$, if there is a set $Y$ such that $Y$ belongs to exactly one of $\operatorname{Mod}_{A}(P)$ and $\operatorname{Mod}_{A}(Q)$, or $Y \in \operatorname{Mod}_{A}(P) \cap \operatorname{Mod}_{A}(Q)$ and $T_{P}(Y) \neq T_{Q}(Y)$, then $Y \backslash T_{P}(Y) \subseteq A$ or $Y \backslash T_{Q}(Y) \subseteq A$. It follows that $Y \subseteq$ At $(P \cup Q) \cup A$. Since for such $Y$ verifying the membership in $\operatorname{Mod}_{A}(P)$ and $\operatorname{Mod}_{A}(Q)$, and testing $T_{P}(Y) \neq T_{Q}(Y)$ can be done in polynomial time in the size of $A t(P \cup Q) \cup A$, our claim and, consequently, the assertion, follows.

(2) Each of these problems reduces to the problem (1) (extend an instance of a problem in (2) with $A$, the set that defines the problem, to specify an instance of the problem (1)). Thus, the bound follows.

(3) and (4) Each of these problems is equivalent to the problem with $B=\emptyset$ and so, can be reduced to the problem (1).

(5) Corollary 3.3 implies that the complement of the problem is in the class NP. Indeed, given two normal programs $P$ and $Q$, if there is a set $Y$ such that (a) $Y$ is a model of exactly one of $P$ and $Q$, or (b) $Y$ is a model of both $P$ and $Q$, and $T_{P}(Y) \neq T_{Q}(Y)$, then there is $Y^{\prime} \subseteq A t(P \cup Q)$ with the same property. Since verifying conditions (a) and (b) for $Y^{\prime} \subseteq A t(P \cup Q)$ can be done in polynomial time in the size of $\bar{P} \cup Q$, our claim and, consequently, the assertion, follows.

In problems (3) - (5) we do not need any explicit or implicit representation of $B$, as the supp-equivalence relative to $\mathcal{H B}^{n}(A, B)$ depends on $A$ only.

We move on to the lower bound (hardness). In several proofs in this and the next sections, we use the following concepts and notation. We consider a CNF formula $\varphi$ over a set of atoms $Y$, or a QBF formula $\forall Y \exists X \varphi$, where $\varphi$ is a CNF formula over the set of atoms $X \cup Y$. For every such atom $z \in Y$ or $z \in X \cup Y$, respectively, we denote by $z^{\prime}$ a new atom not appearing anywhere in $\varphi$, possibly also different from some other atoms that might be named explicitly, and different from other "primed" atoms. Given a set of "non-primed" atoms $Z$, we define $Z^{\prime}=\left\{z^{\prime} \mid z \in Z\right\}$. Finally, for a clause $c=z_{1} \vee \cdots \vee z_{k} \vee \neg z_{k+1} \vee \cdots \vee \neg z_{m}$, we denote by $\hat{c}$ the sequence $z_{1}^{\prime}, \ldots, z_{k}^{\prime}, z_{k+1}, \ldots, z_{m}$.

Theorem 5.2 For every finite $A \subseteq A$ t and $A=A$, and for every $B \subseteq A$, the problem $\operatorname{SUPP}_{A}^{\bar{B}}$ is coNP-hard.

Proof. Let us consider a CNF $\varphi$ and let $Y$ be the set of atoms in $\varphi$. We define

$$
\begin{aligned}
P(\varphi)= & \left\{y \leftarrow \text { not } y^{\prime} ; y^{\prime} \leftarrow \operatorname{not} y \mid y \in Y\right\} \cup \\
& \left\{\leftarrow y, y^{\prime} \mid y \in Y\right\} \cup \\
& \{\leftarrow \hat{c} \mid c \text { is a clause in } \varphi\}
\end{aligned}
$$

To simplify the notation, we write $P$ for $P(\varphi)$. One can check that $\varphi$ has a model if and only if $P$ has a model. Moreover, for every model $M$ of $P$ such that $M \subseteq A t(P)$, 
$M$ is a stable model of $P$. Thus, each such model of $P$ is also a supported model of $P$ and, consequently, satisfies $M=T_{P}(M)$.

Next, we define $Q$ to consist of two rules: $f$ and $\leftarrow$ f. Clearly, $Q$ has no models. By Theorem 3.2, $Q$ is supp-equivalent to $P$ relative to $\mathcal{H B}^{n}(A, B)$ if and only if $\operatorname{Mod}_{A}(Q)=\operatorname{Mod}_{A}(P)$ and for every $M \in \operatorname{Mod}_{A}(Q)$, $T_{Q}(M)=T_{P}(M)$. Since $\operatorname{Mod}_{A}(Q)=\emptyset$, we have that $Q$ is supp-equivalent to $P$ relative to $\mathcal{H B}^{n}(A, B)$ if and only if $\operatorname{Mod}_{A}(P)=\emptyset$. If $M \in \operatorname{Mod}_{A}(P)$, then there is $M^{\prime} \subseteq A t(P)$ such that $M^{\prime} \in \operatorname{Mod}_{A}(P)$. Since every model $M^{\prime}$ of $P$ such that $M^{\prime} \subseteq A t(P)$ satisfies $M^{\prime}=T_{P}\left(M^{\prime}\right)$, it follows that $\operatorname{Mod}_{A}(P)=\emptyset$ if and only if $P$ has no models.

Thus, $\varphi$ is unsatisfiable if and only if $Q$ is supp-equivalent to $P$ relative to $\mathcal{H B}^{n}(A, B)$, and the assertion follows.

We observe that for the result to hold we do not need to know B. Putting together Theorems 5.1 and 5.2 we obtain the following result.

Corollary 5.3 The problems listed in Theorem 5.1 are coNP-complete.

Proof. The hardness of problems in Theorem 5.1 follows from Theorem 5.2. Thus, the coNP-completeness follows.

Problems we considered so far do not impose restrictions on input programs $P$ and $Q$. In particular, they contain instances, in which $\operatorname{Mod}_{A}(P) \neq \operatorname{Mod}_{A}(Q)$, a property exploited by the proof we presented above. We will now consider the problem to decide whether normal programs $P$ and $Q$ such that $\operatorname{Mod}_{A}(P)=\operatorname{Mod}_{A}(Q)$ are supp-equivalent relative to $\mathcal{H B}^{n}(A, B)$. It turns out that this additional information is of no help as the complexity does not go down.

We start with an auxiliary result.

Lemma 5.4 Let $A \subseteq A t$ be a fixed finite non-empty set or $A=A t$. The following problem is coNP-complete. Given a normal logic program $P$, decide whether every $M \in \operatorname{Mod}_{A}(P)$ such that $M \subseteq A t(P)$ is a supported model of $P$.

Proof. Let us select and fix an element in $A$, say $g$, and let $\varphi$ be a CNF formula over $Y$. Wlog we may assume that $\varphi$ does not contain $g$. We define

$$
\begin{aligned}
S(\varphi)= & \left\{y \leftarrow \text { not } y^{\prime} ; y^{\prime} \leftarrow \text { not } y \mid y \in Y\right\} \cup \\
& \left\{\leftarrow y, y^{\prime} \mid y \in Y\right\} \cup \\
& \{g \leftarrow \hat{c} \mid c \text { is a clause in } \varphi\}
\end{aligned}
$$

In the remainder of the proof, we write $S$ for $S(\varphi)$.

We note that for every $M \subseteq A t(S)$, if $M \models S$ then $T_{S}(M)=M$ or $T_{S}(M)=M \backslash\{g\}$. In particular, if $M \subseteq$ $A t(S)$ and $M \models S$, then $M \backslash T_{S}(M) \subseteq\{g\} \subseteq A$. Thus, for $M \subseteq A t(S), M \in \operatorname{Mod}_{A}(S)$ if and only if $\bar{M} \models S$.

If $\varphi$ is unsatisfiable then, for every $M \subseteq A t(S)$ such that $M \models S$, we have $g \in M$. Consequently, each such $M$ is a supported model of $S$. It follows that for every $M \in$ $\operatorname{Mod}_{A}(S)$ such that $M \subseteq A t(S), M$ is a supported model of $S$.

If $\varphi$ is satisfiable, every model of $\varphi$ gives rise to a supported model, say $X$, of $S$, such that $g \notin X$. It is easy to see that $M=X \cup\{g\}$ is a model of $S$ but not a supported one. Since $M \subseteq A t(S)$ and $M$ is a model of $S, M \in \operatorname{Mod}_{A}(S)$.

Thus, $\varphi$ is unsatisfiable if and only if every $M \in$ $\operatorname{Mod}_{A}(S)$ such that $M \subseteq A t(S)$ is a supported model of $S$. Consequently, the hardness follows.

The membership part is evident. Indeed, the complementary problem can be decided by the following algorithm: nondeterministically guess $M \subseteq A t(S)$; verify that (1) $M \in \operatorname{Mod}_{A}(S)$ and that (2) $M$ is not supported model of $S$. Clearly both (1) and (2) can be done in polynomial time (both for $A$ finite and non-empty, and for $A=A t$, where the condition $M \backslash T_{S}(M) \subseteq A$ trivializes). Thus, the complementary problem is in NP and the assertion follows.

Applying Lemma 5.4 to the case $A=A t$, we obtain the following result of some interest in its own right.

Theorem 5.5 The following problem is coNP-complete: given a finite normal logic program $P$, decide whether every model $Y$ of $P$ such that $Y \subseteq A t(P)$ is supported.

However, the primary application of Lemma 5.4 is in determining the complexity of hyperequivalence for programs $P$ and $Q$ with $\operatorname{Mod}_{A}(P)=\operatorname{Mod}_{A}(Q)$, the problem we already mentioned above.

Theorem 5.6 Let $A$ be a fixed finite non-empty subset of At or let $A=A t$. For every set $B \subseteq A$, the following problem is coNP-complete: given two normal programs $P$ and $Q$ such that $\operatorname{Mod}_{A}(P)=\operatorname{Mod}_{A}(Q)$, decide whether they are supp-equivalent relative to $\mathcal{H B}^{n}(A, B)$.

Proof. We restrict to the case $B=\emptyset$ (we recall that suppequivalence does not depend on $B$ ).

The membership part follows from Theorem 5.1. For the hardness part, we will proceed as follows. Given a normal logic program $P$, we will construct a normal logic program $P^{\prime}$ so that (1) $\operatorname{Mod}_{A}(P)=\operatorname{Mod}_{A}\left(P^{\prime}\right)$, and (2) $P$ and $P^{\prime}$ are supp-equivalent relative to $\mathcal{H B}^{n}(A, \emptyset)$ if and only if for every $M \in \operatorname{Mod}_{A}(P)$ such that $M \subseteq A t(P), M$ is a supported model for $P$. Since it will be the case that $P^{\prime}$ can be constructed in polynomial time in the size of $P$ and $A$, the assertion will follow from Lemma 5.4.

Thus, let $P$ be a normal logic program. Let us define $P^{\prime}=P \cup\{g \leftarrow g \mid g \in A t(P) \cap A\}$. It is evident that $P^{\prime}$ can be constructed in polynomial time in the size of $P$ and $A$. We also note that for every $M \subseteq A t(P), T_{P^{\prime}}(M)=$ $T_{P}(M) \cup(M \cap A)$.

We will first prove (1), that is, $\operatorname{Mod}_{A}(P)=\operatorname{Mod}_{A}\left(P^{\prime}\right)$. Let $M \in \operatorname{Mod}_{A}(P)$. Then $M \models P$ (and so, $T_{P}(M) \subseteq M$ ) and $M \backslash T_{P}(M) \subseteq A$. One can verify that $T_{P^{\prime}}(M)=M \cap$ $A t(P)$. Thus, $M \models P^{\prime}$. Moreover, as $T_{P}(M) \subseteq T_{P^{\prime}}(M)$, $M \backslash T_{P^{\prime}}(M) \subseteq A$. Consequently, $M \in \operatorname{Mod}_{A}\left(P^{\prime}\right)$. Conversely, let $M \in \operatorname{Mod}_{A}\left(P^{\prime}\right)$. It follows that $M \models P$. Next, let $y \in M \backslash T_{P}(M)$. If $y \in M \backslash T_{P^{\prime}}(M)$, then $y \in A$ (as $\left.M \backslash T_{P^{\prime}}(M) \subseteq A\right)$. Otherwise, $y \in T_{P^{\prime}}(M) \backslash T_{P}(M)$. It follows that $y \in A \cap A t(P)$ and so, $y \in A$ in this case, too. Thus, we obtain $M \backslash T_{P}(M) \subseteq A$ and, consequently, $M \in \operatorname{Mod}_{A}(P)$. That concludes the proof of $\operatorname{Mod}_{A}(P)=\operatorname{Mod}_{A}\left(P^{\prime}\right)$.

To prove the equivalence (2), let us first assume that for every $M \in \operatorname{Mod}_{A}(P)$ such that $M \subseteq A t(P), M$ is a 
supported model for $P$. We will show that $P$ and $P^{\prime}$ are supp-equivalent relative to $\mathcal{H B}^{n}(A, \emptyset)$. To this end, it suffices to show that for every $M \in \operatorname{Mod}_{A}(P), T_{P}(M)=$ $T_{P^{\prime}}(M)$ (cf. Theorem 3.2). Thus, let $M \in \operatorname{Mod}_{A}(P)$. Then, $M \cap A t(P) \in \operatorname{Mod}_{A}(P)$ and, by the assumption, $T_{P}(M \cap A t(P))=M \cap A t(P)$. Since $T_{P}(M)=T_{P}(M \cap$ $A t(P)), T_{P}(M)=M \cap A t(P)$. We also have $T_{P^{\prime}}(M)=$ $T_{P^{\prime}}(M \cap A t(P))=T_{P}(M \cap A t(P)) \cup[(M \cap A t(P)) \cap A]=$ $M \cap A t(P)$. Thus, $T_{P}(M)=T_{P^{\prime}}(M)$ and so, $P$ and $P^{\prime}$ are supp-equivalent relative to $\mathcal{H B}^{n}(A, \emptyset)$.

Conversely, let $P$ and $P^{\prime}$ be supp-equivalent relative to $\mathcal{H B}^{n}(A, \emptyset)$. Let $M \in \operatorname{Mod}_{A}(P)$ be such that $M \subseteq A t(P)$. We proved earlier that for $M \in \operatorname{Mod}_{A}(P), T_{P^{\prime}}(M)=M \cap$ $A t(P)$. Since $M \subseteq A t(P), T_{P^{\prime}}(M)=M$. Thus, $M$ is a supported model of $P^{\prime}$ and so (as $\emptyset \in \mathcal{H} \mathcal{B}^{n}(A, \emptyset)$ and $P$ and $P^{\prime}$ are supp-equivalent relative to $\mathcal{H} \mathcal{B}^{n}(A, \emptyset)$ ), of $P$, too. Thus, for every $M \in \operatorname{Mod}_{A}(P)$ such that $M \subseteq A t(P)$, $M$ is a supported model of $P$, as required to complete the proof of (2). By our earlier comments, the result follows. $\square$

There seems to be no simple reduction from any problem considered in Corollary 5.3 to the problem from Theorem 5.6 and so, a direct proof is needed. The requirement that $A \neq \emptyset$ is necessary for the complexity result of Theorem 5.6. Indeed, by Corollary 3.4, if $A=\emptyset$, programs $P$ and $Q$ with $\operatorname{Mod}_{A}(P)=\operatorname{Mod}_{A}(Q)$ are necessarily supp-equivalent.

\section{Complexity of Suppmin-Equivalence}

We will use here the same notational schema as in the previous section, but replace supp-equivalence with suppminequivalence and write SUPPMIN instead of SUPP. For instance, we write SUPPMIN ${ }^{B}$ (for $B$ fixed and not part of the input) to denote the following problem: given normal programs $P$ and $Q$, and $A \subseteq A t$, decide whether $P$ and $Q$ are suppmin-equivalent relative to $\mathcal{H B}^{n}(A, B)$.

Deciding suppmin-equivalence relative to $\mathcal{H B}^{n}(A, B)$, where $A=A t$ or $B=A t$, remains in the class coNP and turns out to be coNP-complete. To prove that, we first simplify the conditions for $(X, Y) \in \operatorname{Mod}_{A}^{B}$ if $A=A t$ or $B=A t$.

Lemma 6.1 Let $P$ be a normal logic program and $A, B \subseteq$ At. Then,

1. $(X, Y) \in \operatorname{Mod}_{A t}^{B}(P)$ if and only if the following conditions hold:

(a) $Y \models P$

(b) $X \subseteq Y$

(c) for every $Z \subset Y$, such that $\left.Z\right|_{B}=\left.X\right|_{B}$ and $Z \supseteq X$, $Z \not \models P$

(d) if $\left.X\right|_{B}=\left.Y\right|_{B}$, then $Y \backslash T_{P}(Y) \subseteq X$.

2. $(X, Y) \in \operatorname{Mod}_{A}^{A t}(P)$ if and only if the following conditions hold:

(a) $Y \in \operatorname{Mod}_{A}(P)$

(b) $X \subseteq Y$

(c) If $X \subset Y$ then $X \forall P$.

Proof. If $A=A t$, the condition (1) for $(X, Y) \in \operatorname{Mod}_{A}^{B}(P)$ specializes to (1a) as $Y \in \operatorname{Mod}_{A t}(P)$ if and only if $Y \models P$.
Assuming $B=A t$ has no effect on the condition (1). Thus, it appears without any change as the condition (2a).

Since $A \cup B=A t$, the condition (2) for $(X, Y) \in$ $\operatorname{Mod}_{A}^{B}(P)$ is trivially true, in both cases. For the same reason, the condition (3) for $(X, Y) \in \operatorname{Mod}_{A}^{B}(P)$ specializes to (1b) or (2b), respectively. The conditions (4) and (5) for $(X, Y) \in \operatorname{Mod}_{A}^{B}(P)$ specialize to the conditions (1c) and (1d) in case $A=A t$. If $B=A t$, the condition (4) for $(X, Y) \in \operatorname{Mod}_{A}^{B}(P)$ specializes to (2c). The condition (5) for $(X, Y) \in \operatorname{Mod}_{A}^{B}(P)$ holds true (if $X=Y$ then, trivially, $\left.Y \backslash T_{P}(Y) \subseteq Y\right)$ and can be dropped.

Lemma 6.1 plays a key role in establishing the membership in the class coNP of the relativized suppminequivalence problems for which $A=A t$ or $B=A t$. However, for the case $A=A t$ we need one more important property.

Lemma 6.2 Let $P, Q$ be normal programs and $B \subseteq$ At. If $\operatorname{Mod}_{A t}^{B}(P) \neq \operatorname{Mod}_{A t}^{B}(Q)$, then there is $Y \subseteq A t(P \cup Q) \cup B$ such that $Y$ is a model of exactly one of $P$ and $Q$, or there is $a \in Y$ such that $(Y \backslash\{a\}, Y)$ belongs to exactly one of $\operatorname{Mod}_{A t}^{B}(P)$ and $\operatorname{Mod}_{A t}^{B}(Q)$.

Proof. Let us assume that $P$ and $Q$ have the same models (otherwise, there is $Y \subseteq A t(P \cup Q)$ that is a model of exactly one of $P$ and $Q$, and the assertion follows). Wlog we can assume that there is $(X, Y) \in \operatorname{Mod}_{A t}^{B}(P) \backslash \operatorname{Mod}_{A t}^{B}(Q)$. Moreover, we can assume that $Y \subseteq A t(P \cup Q) \cup B$. It follows that $(X, Y)$ satisfies the conditions (1a)-(1d) from Lemma 6.1 for $(X, Y) \in \operatorname{Mod}_{A t}^{B}(P)$. Moreover, since $P$ and $Q$ have the same models, $(X, Y)$ already satisfies conditions (1a)-(1c) for $(X, Y) \in \operatorname{Mod}_{A t}^{B}(Q)$. Hence $\left.X\right|_{B}=$ $\left.Y\right|_{B}$ and $Y \backslash T_{Q}(Y) \nsubseteq X$ have to hold. Thus, there is $a \in\left(Y \backslash T_{Q}(Y)\right) \backslash X$. We will show that $(Y \backslash\{a\}, Y) \in$ $\operatorname{Mod}_{A t}^{B}(P)$ and $(Y \backslash\{a\}, Y) \notin \operatorname{Mod}_{A t}^{B}(Q)$.

Since $(X, Y) \in \operatorname{Mod}_{A t}^{B}(P), Y$ is a model of $P$. Next, obviously, $Y \backslash\{a\} \subseteq Y$. Thus, the conditions (1a) and (1b) of Lemma 6.1 hold. Let $Z \subset Y$ be such that $Z \supseteq Y \backslash\{a\}$. Then $Z=Y \backslash\{a\}$. We have $\left.Y\right|_{B}=\left.X\right|_{B}, a \in Y$, and $a \notin X$. Thus, $a \notin B$. It follows that $\left.(Y \backslash\{a\})\right|_{B}=\left.X\right|_{B}$ and $X \subseteq Y \backslash\{a\}$. Since $(X, Y) \in \operatorname{Mod}_{A t}^{B}(P), Y \backslash\{a\} \not \models P$, that is, $Z \not \models P$. Thus, the condition (1c) of Lemma 6.1 holds.

Since $a \notin B,\left.(Y \backslash\{a\})\right|_{B}=\left.Y\right|_{B}$. Thus, we also have to verify the condition (1d) of Lemma 6.1. We have $Y \backslash T_{P}(Y) \subseteq X$ (we recall that $\left.\left.Y\right|_{B}=\left.X\right|_{B}\right)$ and so, $Y \backslash$ $T_{P}(Y) \subseteq Y \backslash\{a\}$. Hence, the condition (1d) of Lemma 6.1 holds, for $P$ and, consequently, $(Y \backslash\{a\}, Y) \in \operatorname{Mod}_{A t}^{B}(P)$. On the other hand, $a \in Y \backslash T_{Q}(Y)$ and $a \notin Y \backslash\{a\}$. Thus, the condition (1d) of Lemma 6.1 does not hold for $Q$ and so, $(Y \backslash\{a\}, Y) \notin \operatorname{Mod}_{A t}^{B}(Q)$.

We are now ready to show the promised coNPcompleteness results.

Theorem 6.3 The following problems are coNP-complete:

1. SUPPMIN ${ }_{A t}^{B}$, for every finite $B \subseteq A t$,

2. $\operatorname{SUPPMIN}_{A}^{A t}$, for every finite $A \subseteq A t$, 


\section{3. $\operatorname{SUPPMIN}^{A t}, \operatorname{SUPPMIN}_{A t}$, and $\operatorname{SUPPMIN}_{A t}^{A t}$.}

Proof. The case of SUPPMIN ${ }_{A t}^{A t}$ is already clear by Corollary 4.8 and Theorem 5.2.

To establish the other cases, we will show coNPmembership for SUPPMIN $^{A t}$ and SUPPMIN $A t$. From these two results, the membership results for $\operatorname{SUPPMIN}_{A t}^{B}$ and SUPPMIN $_{A}^{A t}$ (for finite $A, B \subseteq A t$ ) follow easily.

Likewise, we will show coNP-hardness for SUPPMIN ${ }_{A t}^{B}$ and $\operatorname{SuPPMIN}_{A}^{A t}$ (for finite $A, B \subseteq A t$ ). That implies the corresponding lower bounds for SUPPMIN ${ }^{A t}$ and SUPPMIN $_{A t}$.

We start with coNP-membership for $\operatorname{SUPPMIN}_{A t}$. The following nondeterministic algorithm verifies, given programs $P, Q$ and $B \subseteq A t$, that $P$ and $Q$ are not suppminequivalent relative to $\mathcal{H} \mathcal{B}^{n}(A t, B)$. We guess a pair $(a, Y)$, where $Y \subseteq A t(P \cup Q) \cup B$, and $a \in A t$ such that (1) $Y$ is a model of exactly one of $P$ and $Q$; or (2) $a \in Y$ and $(Y \backslash\{a\}, Y)$ belongs to exactly one of $\operatorname{Mod}_{A t}^{B}(P)$ and $\operatorname{Mod}_{A t}^{B}(Q)$; or (3) $Y$ is model of both $P$ and $Q$ and $\left.T_{P}(Y)\right|_{B} \neq\left. T_{Q}(Y)\right|_{B}$.

Such a pair exists if and only if $P$ and $Q$ are not suppminequivalent relative to $\mathcal{H B}^{n}(A t, B)$. Indeed, let us assume that such a pair $(a, Y)$ exists. If (1) holds for $(a, Y)$, say $Y$ is a model of $P$ but not $Q$, then $(Y, Y) \in \operatorname{Mod}_{A t}^{B}(P) \backslash$ $\operatorname{Mod}_{A t}^{B}(Q)$ (easy to verify by means of Lemma 6.1). Thus, $\operatorname{Mod}_{A t}^{B}(P) \neq \operatorname{Mod}_{A t}^{B}(Q)$ and, by Theorem 4.6, $P$ and $Q$ are not suppmin-equivalent relative to $\mathcal{H B}^{n}(A t, B)$. If (2) holds for $(a, Y), \operatorname{Mod}_{A t}^{B}(P) \neq \operatorname{Mod}_{A t}^{B}(Q)$ again, and we reason as above. Finally, if neither (1) nor (2) holds, Lemma 6.2 implies $\operatorname{Mod}_{A t}^{B}(P)=\operatorname{Mod}_{A t}^{B}(Q)$. In this case, (3) holds for $(a, Y)$. Since $Y \models P$, we have $(Y, Y) \in \operatorname{Mod}_{A t}^{B}(P)$. Moreover, $\left.T_{P}(Y)\right|_{B} \neq\left. T_{Q}(Y)\right|_{B}$. Thus, again by Theorem 4.6, $P$ and $Q$ are not suppmin-equivalent relative to $\mathcal{H B}^{n}(A t, B)$.

Conversely, if $P$ and $Q$ are not suppmin-equivalent relative to $\mathcal{H B}^{n}(A t, B)$, then $\operatorname{Mod}_{A t}^{B}(P) \neq \operatorname{Mod}_{A t}^{B}(Q)$ or there is $(X, Y) \in \operatorname{Mod}_{A t}^{B}(P)$ such that $\left.T_{P}(Y)\right|_{B} \neq\left. T_{Q}(Y)\right|_{B}$. The former implies (by Lemma 6.2) that there is $(a, Y)$, with $Y \subseteq A t(P \cup Q) \cup B)$, that satisfies (1) or (2). Thus, let us assume that $\operatorname{Mod}_{A t}^{B}(P)=\operatorname{Mod}_{A t}^{B}(Q)$ and that there is $(X, Y) \in \operatorname{Mod}_{A t}^{B}(P)$ such that $\left.T_{P}(Y)\right|_{B} \neq\left. T_{Q}(Y)\right|_{B}$. Since $(X, Y) \in \operatorname{Mod}_{A t}^{B}(Q)$, too, $Y$ is a model of both $P$ and $Q$ and $\left.T_{P}(Y)\right|_{B} \neq\left. T_{Q}(Y)\right|_{B}$. Clearly, $Y^{\prime}=Y \cap$ $(A t(P \cup Q) \cup B)$ is a model of both $P$ and $Q$, too, and $\left.T_{P}\left(Y^{\prime}\right)\right|_{B} \neq\left. T_{Q}\left(Y^{\prime}\right)\right|_{B}$. Picking any $a \in A t$ yields a pair $\left(a, Y^{\prime}\right)$, with $Y^{\prime} \subseteq A t(P \cup Q) \cup B$, for which (3) holds.

It follows that the algorithm is correct. Moreover, checking whether $Y \models P$ and $Y \models Q$ can clearly be done in polynomial time in the total size of $P, Q$, and $B$; the same holds for checking $\left.T_{P}(Y)\right|_{B} \neq\left. T_{Q}(Y)\right|_{B}$. Finally, by Lemma 6.1, testing $(Y \backslash\{a\}, Y) \in \operatorname{Mod}_{A t}^{B}(P)$ and $(Y \backslash\{a\}, Y) \in \operatorname{Mod}_{A t}^{B}(Q)$ are polynomial-time (with respect to the size of the input) tasks, too; the only problematic condition is (1c) from Lemma 6.1. However, we need to test that $Z \forall P$ there for only one $Z$ such that $Y \backslash\{a\} \subseteq Z \subset Y$, namely $Z=Y \backslash\{a\}$. Thus, the algo- rithm runs in polynomial time. It follows that the complement of our problem is in the class NP and so the assertion follows.

We continue by showing that SUPPMIN ${ }^{A t}$ is in the class coNP. By Theorem 4.6, $P$ and $Q$ are suppmin-equivalent relative to $\mathcal{H B}^{n}(A, A t)$ if and only if $\operatorname{Mod}_{A}^{A t}(P)=$ $\operatorname{Mod}_{A}^{A t}(Q)$ and for every $(X, Y) \in \operatorname{Mod}_{A}^{A t}(Y), T_{P}(Y)=$ $T_{Q}(Y)$. It follows that to decide that $P$ and $Q$ are not suppmin-equivalent relative to $\mathcal{H} \mathcal{B}^{n}(A, A t)$, it suffices to guess a pair $(X, Y)$, where $X \subseteq Y \subseteq A t(P \cup Q) \cup$ $A$, and verify that (a) $(X, Y)$ belongs to exactly one of $\operatorname{Mod}_{A}^{A t}(P)$ and $\operatorname{Mod}_{A}^{A t}(P)$, or (b) that $(X, Y)$ belongs to both $\operatorname{Mod}_{A}^{A t}(P)$ and $\operatorname{Mod}_{A}^{A t}(P)$, and $T_{P}(Y) \neq T_{Q}(Y)$. Indeed, if $(X, Y) \in \operatorname{Mod}_{A}^{A t}(P) \cup \operatorname{Mod}_{A}^{A t}(Q)$, then $X \subseteq Y \subseteq$ $A t(P \cup Q) \cup A$ (the latter inclusion following from the fact that $\left.Y \in \operatorname{Mod}_{A}(P) \cup \operatorname{Mod}_{A}(Q)\right)$. By Lemma 6.1, and since $X \subseteq Y \subseteq A t(P \cup Q) \cup A$, all these tests can be executed in polynomial time in the total size of $P, Q$ and $A$. Thus, the complementary problem is in NP and so, the membership in coNP follows.

We now switch over to the hardness results and start this time with SUPPMIN $A t$. In the proof of Theorem 5.6, we have shown that for every program $P$, and any finite $A \subseteq A t$, $\operatorname{Mod}_{A}(P)=\operatorname{Mod}_{A}\left(P^{\prime}\right)$, where $P^{\prime}=P \cup\{g \leftarrow g \mid g \in$ $A \cap A t(P)\}$. Moreover, in the same proof, we have shown that $P$ and $P^{\prime}$ are supp-equivalent relative to $\mathcal{H B}^{n}(A, A t)$ if and only if for each $Y \in \operatorname{Mod}_{A}(P)$ with $Y \subseteq A t(P), Y$ is a supported model of $P$. We will now show that $P$ and $P^{\prime}$ are supp-equivalent relative to $\mathcal{H} \mathcal{B}^{n}(A, A t)$, if and only if $P$ and $P^{\prime}$ are suppmin-equivalent relative to $\mathcal{H B}^{n}(A, A t)$. The only-if direction follows from the fact that $P$ and $P^{\prime}$ have the same models. Indeed, this property implies that for every $R \in \mathcal{H B}^{n}(A, A t), P \cup R$ and $Q \cup R$ have the same supported models and the same models. Consequently, they have the same supported minimal models.

For the if-direction, we recall that suppmin-equivalence relative to $\mathcal{H} \mathcal{B}^{n}(A, A t)$ between $P$ and $P^{\prime}$ implies (by Theorem 4.6) $\operatorname{Mod}_{A}^{A t}(P)=\operatorname{Mod}_{A}^{A t}\left(P^{\prime}\right)$ and $T_{P}(Y)=T_{P^{\prime}}(Y)$, for every $(X, Y) \in \operatorname{Mod}_{A}^{A t}(P)$. In view of Lemma 6.1, it is easy to see that $\operatorname{Mod}_{A}(P)=\operatorname{Mod}_{A}\left(P^{\prime}\right)$ follows. Moreover, by the same lemma, if $Y \in \operatorname{Mod}_{A}(P)$ then $(Y, Y) \in \operatorname{Mod}_{A}^{A t}(P)$. Thus, $T_{P}(Y)=T_{P^{\prime}}(Y)$, for every $Y \in \operatorname{Mod}_{A}(P)$. By Theorem 3.2, $P$ and $P^{\prime}$ are suppequivalent relative to $\mathcal{H B}^{n}(A, A t)$.

It follows that for every $Y \in \operatorname{Mod}_{A}(P)$ with $Y \subseteq A t(P)$, $Y$ is a supported model for $P$ if and only if $P$ and $P^{\prime}$ are suppmin-equivalent relative to $\mathcal{H} \mathcal{B}^{n}(A, A t)$. Thus, the assertion follows from Lemma 5.4.

To prove the coNP-hardness of SUPPMIN ${ }_{A t}^{B}$, we proceed as follows. Let $\varphi$ be a CNF formula, and let $Y$ be the set of atoms in $\varphi$. We define $P$ and $Q$ as in the proof of Theorem 5.2. Since $Q$ has no models, $\operatorname{Mod}_{A t}^{B}(Q)=\emptyset$.

Let $Y$ be a model of $P$. Clearly, $(Y, Y)$ satisfies the conditions (1a)-(1d) from Lemma 6.1. Thus, $\operatorname{Mod}_{A t}^{B}(P) \neq \emptyset$. Conversely, if $\operatorname{Mod}_{A t}^{B}(P) \neq \emptyset$, then there is $(X, Y) \in$ $\operatorname{Mod}_{A t}^{B}(P)$. By Lemma 6.1(1a), $Y$ is a model of $P$.

Thus, $P$ has models if and only if $\operatorname{Mod}_{A t}^{B}(P) \neq \emptyset$. By 
Theorem 4.6, $P$ has models if and only if $P$ and $Q$ are not suppmin-equivalent relative to $\mathcal{H B}^{n}(A t, B)$.

In the proof of Theorem 5.2, we already showed that $P$ has models if and only if $\varphi$ has models. Thus, $\varphi$ has models if and only if $P$ and $Q$ are not suppmin-equivalent relative to $\mathcal{H B}^{n}(A t, B)$. Consequently, the claim follows.

We will now establish the complexity of deciding relativized suppmin-equivalence when $A$ and $B$ are finite (fixed as part of the problem specification, or given as part of instance specification). We start with an auxiliary result needed to derive upper bounds for the complexity.

Lemma 6.4 The following problem is in coNP: given a normal logic program $P$ and sets $X, Y, A, B \subseteq A$, decide whether $(X, Y) \in \operatorname{Mod}_{A}^{B}(P)$.

Proof. We already established earlier that deciding whether $Y \notin \operatorname{Mod}_{A}(P)$ (condition (1)) can be done in polynomial time in the size of $P, Y$ and $A$. The same is evident for deciding $\left.X \nsubseteq Y\right|_{A \cup B}$ (condition (3)) and $Y \backslash T_{P}(Y) \nsubseteq X$, in case, $\left.X\right|_{B}=\left.Y\right|_{B}$ (condition (5)).

The remaining two conditions defining $(X, Y) \in$ $\operatorname{Mod}_{A}^{B}(P)$, that is, (2) and (4), can be checked for violation as follows. We guess $Z \subset Y$ such that either $\left.Z\right|_{A \cup B}=$ $\left.Y\right|_{A \cup B}$, or jointly $\left.Z\right|_{B}=\left.X\right|_{B}$ and $\left.\left.Z\right|_{A} \supseteq X\right|_{A}$. Then, we check whether $Z \models P$. Thus, deciding whether $(X, Y) \notin$ $\operatorname{Mod}_{A}^{B}(P)$, for given sets $X, Y, A, B \subseteq A t$, is in the class NP. Consequently, deciding whether $(X, Y) \in \operatorname{Mod}_{A}^{B}(P)$, for given sets $X, Y, A, B \subseteq A t$, is in the class coNP.

With this result in hand, $\Pi_{2}^{P}$-membership of SUPPMIN can be shown by suitably guessing pairs $(X, Y)$ in $\operatorname{Mod}_{A}^{B}(P)$ and $\operatorname{Mod}_{A}^{B}(P)$, respectively.

Theorem 6.5 The problem SUPPMIN is in $\Pi_{2}^{P}$.

Proof. The complementary problem can be decided in nondeterministic polynomial time with an access to an NPoracle. Indeed, we note that if $(X, Y) \in \operatorname{Mod}_{A}^{B}(P)$, then $Y \subseteq A t(P) \cup A$. Thus, if there exists $(X, Y)$ that belongs to exactly one of $\operatorname{Mod}_{A}^{B}(P)$ and $\operatorname{Mod}_{A}^{B}(Q)$, then there is $\left(X^{\prime}, Y^{\prime}\right)$ with that property and such that $Y^{\prime} \subseteq$ $A t(P \cup Q) \cup A$. Moreover, if $\operatorname{Mod}_{A}^{B}(P)=\operatorname{Mod}_{A}^{B}(Q)$ and there is $(X, Y) \in \operatorname{Mod}_{A}^{B}(P)$ such that $\left.T_{P}(Y)\right|_{B} \neq$ $\left.T_{Q}(Y)\right|_{B}$, then there is $\left(X^{\prime}, Y^{\prime}\right) \in \operatorname{Mod}_{A}^{B}(P)$ such that $Y^{\prime} \subseteq A t(P \cup Q) \cup A$ and $\left.T_{P}\left(Y^{\prime}\right)\right|_{B} \neq\left. T_{Q}\left(Y^{\prime}\right)\right|_{B}$. Thus, to decide the complementary problem, it suffices to guess sets $X, Y \subseteq A t(P \cup Q) \cup A$ and check that $(X, Y)$ is in exactly one of $\operatorname{Mod}_{A}^{B}(P)$ and in $\operatorname{Mod}_{A}^{B}(Q)$, or that $(X, Y)$ is in both $\operatorname{Mod}_{A}^{B}(P)$ and $\operatorname{Mod}_{A}^{B}(Q)$, and $\left.T_{P}(Y)\right|_{B} \neq\left. T_{Q}(Y)\right|_{B}$. By Lemma 6.4, the tests for the membership in $\operatorname{Mod}_{A}^{B}(P)$ and $\operatorname{Mod}_{A}^{B}(Q)$ can be accomplished by an NP-oracle and all other tasks are evidently in the class P.

We show the matching lower bound for the more specialized problem SUPPMIN $A$.

Theorem 6.6 The problem $\operatorname{SUPPMIN}_{B}^{A}$ is $\Pi_{2}^{P}$-hard, for every finite $A, B \subseteq A t$.
Proof. Let $\forall Y \exists X \varphi$ be a QBF, where $\varphi$ is a CNF formula over $X \cup Y$. We can assume that $(A \cup B) \cap X=\emptyset$ (if not, variables in $X$ can be renamed). Next, we can assume that $A, B \subseteq Y$. Indeed, $\varphi^{+}$obtained by expanding $\varphi$ with clauses $z \vee \neg z$, for each $z \in A \cup B$, has the property that $\forall Y \exists X \varphi$ is true if and only if $\forall Y^{+} \exists X \varphi^{+}$is true, where $Y^{+}=Y \cup A \cup B$.

We will construct programs $P(\varphi)$ and $Q(\varphi)$ so that $\forall Y \exists X \varphi$ is true if and only if $P(\varphi)$ and $Q(\varphi)$ are suppminequivalent relative to $\mathcal{H B}^{n}(A, B)$. Since it will be possible to implement the construction to run in polynomial time in the size of $\varphi$, and since the problem to decide whether a given QBF $\forall Y \exists X \varphi$ is true is $\Pi_{2}^{P}$-complete, the assertion will follow.

We use priming and $\hat{c}$ as discussed above and define the following programs:

$$
\begin{aligned}
P(\varphi)= & \left\{z \leftarrow \text { not } z^{\prime} ; z^{\prime} \leftarrow \text { not } z \mid z \in X \cup Y\right\} \cup \\
& \left\{\leftarrow y, y^{\prime} \mid y \in Y\right\} \cup \\
& \left\{x \leftarrow u, u^{\prime} ; x^{\prime} \leftarrow u, u^{\prime} \mid x, u \in X\right\} \cup \\
& \left\{x \leftarrow \hat{c} ; x^{\prime} \leftarrow \hat{c} \mid x \in X, c \text { is a clause in } \varphi\right\} ; \\
Q(\varphi)= & \left\{z \leftarrow \text { not } z^{\prime} ; z^{\prime} \leftarrow \text { not } z \mid z \in X \cup Y\right\} \cup \\
& \left\{\leftarrow z, z^{\prime} \mid z \in X \cup Y\right\} \cup \\
& \{\leftarrow \hat{c} \mid c \text { is a clause in } \varphi\} .
\end{aligned}
$$

To simplify notation, from now on we write $P$ for $P(\varphi)$ and $Q$ for $Q(\varphi)$. We observe that $A t(P)=A t(Q)=W$, where $W=X \cup X^{\prime} \cup Y \cup Y^{\prime}$.

One can check that the models of $Q$ contained in $W$ are sets

$$
I \cup(Y \backslash I)^{\prime} \cup J \cup(X \backslash J)^{\prime}
$$

where $J \subseteq X, I \subseteq Y$ and $I \cup J \models \varphi$. Each model of $Q$ is also a model of $P$ but $P$ has additional models contained in $W$. They are of the form:

$$
I \cup(Y \backslash I)^{\prime} \cup X \cup X^{\prime},
$$

for each $I \subseteq Y$. Clearly, for each model $M$ of $Q$ such that $M \subseteq W, T_{Q}(M)=M$. Similarly, for each model $M$ of $P$ such that $M \subseteq W, T_{P}(M)=M$.

From these comments, it follows that for every model $M$ of $Q$ (resp. $P$ ), $T_{Q}(M)=M \cap W$ (resp. $T_{P}(M)=M \cap$ $W$ ). Since $B \subseteq W$, for every model $M$ of both $P$ and $Q$, $\left.T_{Q}(M)\right|_{B}=M \cap W \cap B=\left.T_{P}(M)\right|_{B}$. Thus, $P$ and $Q$ are suppmin-equivalent if and only if $\operatorname{Mod}_{A}^{B}(P)=\operatorname{Mod}_{A}^{B}(Q)$ (indeed, we recall that if $(N, M) \in \operatorname{Mod}_{A}^{B}(R)$ then $M$ is a model of $R$ ).

Let us assume that $\forall Y \exists X \varphi$ is false. We will first show that $\operatorname{Mod}_{A}^{B}(P) \neq \operatorname{Mod}_{A}^{B}(Q)$, that is, $P$ and $Q$ are not suppmin-equivalent relative to $\mathcal{H B}^{n}(A, B)$. Since $\forall Y \exists X \varphi$ is false, there exists an assignment $I \subseteq Y$ to atoms $Y$ such that for every $J \subseteq X, I \cup J \not \neq \varphi$. Let $N=$ $I \cup(Y \backslash I)^{\prime} \cup X \cup X^{\prime}$.

We note that $\left(\left.N\right|_{A \cup B}, N\right) \in \operatorname{Mod}_{A}^{B}(P)$. Indeed, since $N$ is a supported model of $P, N \in \operatorname{Mod}_{A}(P)$. The requirement (3) for $\left(\left.N\right|_{A \cup B}, N\right) \in \operatorname{Mod}_{A}^{B}(P)$ is evident. The requirement (5) holds, since $N \backslash T_{P}(N)=\emptyset$. By the property of $I, N$ is a minimal model of $P$. Thus, the requirements 
(2) and (4) hold, too, and $\left(\left.N\right|_{A \cup B}, N\right) \in \operatorname{Mod}_{A}^{B}(P)$ follows. However, since $N$ is not a model of $Q,\left(\left.N\right|_{A \cup B}, N\right) \notin$ $\operatorname{Mod}_{A}^{B}(Q)$. Thus, $\operatorname{Mod}_{A}^{B}(P) \neq \operatorname{Mod}_{A}^{B}(Q)$ and $P$ and $Q$ are not suppmin-equivalent relative to $\mathcal{H}^{n}(A, B)$, as claimed.

Let us assume now that $\forall Y \exists X \varphi$ is true. We will show that $\operatorname{Mod}_{A}^{B}(P)=\operatorname{Mod}_{A}^{B}(Q)$, that is, $P$ and $Q$ are suppminequivalent relative to $\mathcal{H}^{n}(A, B)$. First, we observe that $\operatorname{Mod}_{A}^{B}(Q) \subseteq \operatorname{Mod}_{A}^{B}(P)$. Indeed, let $(M, N) \in \operatorname{Mod}_{A}^{B}(Q)$. It follows that $N$ is a model of $Q$ and, consequently, of $P$. From our earlier comments, it follows that $T_{Q}(N)=$ $T_{P}(N)$. Since $N \backslash T_{Q}(N) \subseteq A, N \backslash T_{P}(N) \subseteq A$. Thus, $N \in \operatorname{Mod}_{A}(P)$. Moreover, if $\left.M\right|_{B}=\left.N\right|_{B}$ then $N \backslash T_{Q}(N) \subseteq M$ and, consequently, $N \backslash T_{P}(N) \subseteq M$. Thus, the requirement (5) for $(M, N) \in \operatorname{Mod}_{A}^{B}(P)$ holds. The condition $\left.M \subseteq N\right|_{A \cup B}$ is evident (it holds as $(M, N) \in$ $\left.\operatorname{Mod}_{A}^{B}(Q)\right)$. Since $N$ is a model of $Q, N=N^{\prime} \cup V$, where $N^{\prime}$ is of the form (1) and $V \subseteq A t \backslash W$. Thus, every model $Z \subset N$ of $P$ is also a model of $Q$. It implies that the requirements (2) and (4) for $(M, N) \in \operatorname{Mod}_{A}^{B}(P)$ hold. Hence, $(M, N) \in \operatorname{Mod}_{A}^{B}(P)$ and, consequently, $\operatorname{Mod}_{A}^{B}(Q) \subseteq \operatorname{Mod}_{A}^{B}(P)$.

The assumption that $\forall Y \exists X \varphi$ is true is needed to prove the converse inclusion. Let $(M, N) \in \operatorname{Mod}_{A}^{B}(P)$. If $N=$ $N^{\prime} \cup V$, where $N^{\prime}$ is of the form (1) and $V \subseteq A t \backslash W$, then arguing as above, one can show that $(M, N) \in \operatorname{Mod}_{A}^{B}(Q)$. Therefore, let us assume that $N=N^{\prime} \cup V$, where $N^{\prime}$ is of the form (2) and $V \subseteq A t \backslash W$. More specifically, let $N^{\prime}=I \cup(Y \backslash I)^{\prime} \cup X \cup X^{\prime}$. By our assumption, there is $J \subseteq X$ such that $I \cup J \models \varphi$. That is, $Z=I \cup(Y \backslash I)^{\prime} \cup$ $J \cup(X \backslash J)^{\prime}$ is a model of $P$. Clearly, $Z \subset N$. Moreover, since $A, B \subseteq Y$, it follows that $\left.Z\right|_{A \cup B}=\left.N\right|_{A \cup B}$. Since $(M, N) \in \operatorname{Mod}_{A}^{B}(P)$, the requirement (2) implies that $Z$ is not a model of $P$, a contradiction. Hence, the latter case is impossible and $\operatorname{Mod}_{A}^{B}(P) \subseteq \operatorname{Mod}_{A}^{B}(Q)$ follows. Consequently, $\operatorname{Mod}_{A}^{B}(P)=\operatorname{Mod}_{A}^{B}(Q)$, that is, $P$ and $Q$ are suppmin-equivalent relative to $\mathcal{H} \mathcal{B}^{n}(A, B)$.

We proved that $\forall Y \exists X \varphi$ is true if and only if $\operatorname{Mod}_{A}^{B}(P)=$ $\operatorname{Mod}_{A}^{B}(Q)$. This completes the proof of the assertion.

Putting together Theorems 6.5 and 6.6 yields the following corollary.

\section{Theorem 6.7 The following problems are $\Pi_{2}^{P}$-complete:}

\section{SUPPMIN}

2. SUPPMIN ${ }^{B}, \operatorname{SUPPMIN}_{A}, \operatorname{SUPPMIN}_{A}^{B}$, for every finite $A, B \subseteq A t$.

Similarly as for supp-equivalence, having additional information that sets $\operatorname{Mod}_{A}^{B}(P)$ and $\operatorname{Mod}_{A}^{B}(Q)$ coincide does not make the problem of deciding suppmin-equivalence easier.

Theorem 6.8 Let $A, B \subseteq$ At be finite and such that $A \cap$ $B \neq \emptyset$. The following problem is $\Pi_{2}^{P}$-complete: given normal programs $P, Q$ such that $\operatorname{Mod}_{A}^{B}(P)=\operatorname{Mod}_{A}^{B}(Q)$, decide whether $P$ and $Q$ are suppmin-equivalent relative to $\mathcal{H B}^{n}(A, B)$.
Proof. The problem reduces to the one considered in Theorem 6.5. Thus, it belongs to $\Pi_{2}^{P}$. To prove $\Pi_{2}^{P}$-hardness we proceed as follows. Let $\forall Y \exists X \varphi$ be a QBF, where $\varphi$ is a CNF over $X \cup Y$. We can assume that $(A \cup B) \cap(X \cup Y)=\emptyset$ (as we can always rename variables in $\varphi$ ). We also choose and fix an element $g \in A \cap B$.

We use priming and $\hat{c}$ as before, and select an atom $x_{0} \in$ $X$. We define the following programs $P(\varphi)$ and $Q(\varphi)$ :

$$
\begin{aligned}
P(\varphi)= & \left\{z \leftarrow \text { not } z^{\prime} ; z^{\prime} \leftarrow \text { not } z \mid z \in X \cup Y\right\} \cup \\
& \left\{\leftarrow y, y^{\prime} \mid y \in Y\right\} \cup \\
& \left\{x \leftarrow u, u^{\prime} ; x^{\prime} \leftarrow u, u^{\prime} \mid x, u \in X\right\} \cup \\
& \left\{x \leftarrow \hat{c} ; x^{\prime} \leftarrow \hat{c} \mid x \in X, c \text { is a clause in } \varphi\right\} \cup \\
& \left\{\leftarrow \text { not } g ; g \leftarrow x_{0}, \text { not } x_{0}^{\prime} ; g \leftarrow x_{0}^{\prime}, \text { not } x_{0}\right\} ; \\
Q(\varphi)= & P(\varphi) \cup\left\{g \leftarrow x_{0}, x_{0}^{\prime}\right\} ;
\end{aligned}
$$

Clearly, the programs $P(\varphi)$ and $Q(\varphi)$ can be constructed in polynomial time in the size of $\varphi$. To simplify notation, from now on we write $P$ for $P(\varphi)$ and $Q$ for $Q(\varphi)$. We set $W=X \cup X^{\prime} \cup Y \cup Y^{\prime} \cup\{g\}$.

We will first prove that $P$ and $Q$ form an instance to the problem in question, that is, $\operatorname{Mod}_{A}^{B}(P)=\operatorname{Mod}_{A}^{B}(Q)$. We will then show that $\forall Y \exists X \varphi$ is true if and only if $P$ and $Q$ are suppmin-equivalent relative to $\mathcal{H B}^{n}(A, B)$. That will imply the $\Pi_{2}^{P}$-hardness of the problem considered in the assertion and will complete the proof.

Clearly, every model of $P$ contains $g$. It follows that $P$ and $Q$ have the same models. To describe them, we first observe that every model of $P$ (and $Q$ ) contained in $W$ is of one of the following two types:

1. $\{g\} \cup I \cup(Y \backslash I)^{\prime} \cup J \cup(X \backslash J)^{\prime}$, for each $I \subseteq Y$ and $J \subseteq X$ such that $I \cup J \models \varphi$;

2. $\{g\} \cup I \cup(Y \backslash I)^{\prime} \cup X \cup X^{\prime}$, for each $I \subseteq Y$.

Thus, every model of $P$ (and of $Q$ ) is of the form $N \cup S$, where $N \subseteq W$ is of type 1 or type 2 , above, and $S \subseteq A t \backslash$ $W$. We refer to $N$ as the $W$-core of the model $N \cup S$. We refer to a model of $P$ (and $Q$ ) as type 1 or type 2, according to the form of its $W$-core.

Next, we observe that for every $N \subseteq A t, T_{P}(N) \subseteq$ $T_{Q}(N)$ and $T_{Q}(N) \backslash T_{P}(N) \subseteq\{g\}$. Let $N \in \operatorname{Mod}_{A}(P)$. It follows that $N$ is a model of $P$ and so, of $Q$, too. We also have $N \backslash T_{Q}(N) \subseteq N \backslash T_{P}(N) \subseteq A$. It follows that $N \in \operatorname{Mod}_{A}(Q)$. Conversely, let $N \in \operatorname{Mod}_{A}(Q)$. Then, $N \models Q$ and so, $N \models P$, Moreover, $N \backslash T_{P}(N) \subseteq$ $\left(N \backslash T_{Q}(N)\right) \cup\{g\} \subseteq A \cup\{g\}=A$. Thus, $N \in \operatorname{Mod}_{A}(P)$. It follows that $\operatorname{Mod}_{A}(P)=\operatorname{Mod}_{A}(Q)$.

Let $(M, N) \in \operatorname{Mod}_{A}^{B}(P)$. Then $N \in \operatorname{Mod}_{A}(P)$ and so, $N \in \operatorname{Mod}_{A}(Q)$. We also have $\left.M \subseteq N\right|_{A \cup B}$. Thus, the conditions (1) and (3) required for $(M, N) \in \operatorname{Mod}_{A}^{B}(Q)$ hold. The conditions (2) and (4) for $(M, N) \in \operatorname{Mod}_{A}^{B}(Q)$ hold as they hold for $P$, and $P$ and $Q$ have the same models. Finally, the condition (5) for $(M, N) \in \operatorname{Mod}_{A}^{B}(Q)$ holds, too, as $N \backslash T_{Q}(N) \subseteq N \backslash T_{P}(N)$. Thus, $\operatorname{Mod}_{A}^{B}(P) \subseteq$ $\operatorname{Mod}_{A}^{B}(Q)$.

Conversely, let $(M, N) \in \operatorname{Mod}_{A}^{B}(Q)$. Reasoning as above, we show that the conditions (1)-(4) for $(M, N) \in$ 
$\operatorname{Mod}_{A}^{B}(P)$ hold. To prove the condition (5), let us assume that $\left.N\right|_{B}=\left.M\right|_{B}$. Since $N \in \operatorname{Mod}_{A}(Q), N$ is a model of $Q$, and thus $g \in N$. Moreover, since $g \in B, g \in M$ as well. We have $N \backslash T_{Q}(N) \subseteq M$. Thus, $N \backslash T_{P}(N) \subseteq M$ follows from our previous observations. Consequently, the condition (5) for $(M, N) \in \operatorname{Mod}_{A}^{B}(P)$ holds, and the inclusion $\operatorname{Mod}_{A}^{B}(Q) \subseteq \operatorname{Mod}_{A}^{B}(P)$ follows.

Thus, $\operatorname{Mod}_{A}^{B}(P)=\operatorname{Mod}_{A}^{B}(Q)$ and so, $P$ and $Q$ form a valid instance to the problem we are considering. Following the proof outline given above, we will now show that $\forall Y \exists X \varphi$ is true if and only if $P$ and $Q$ are suppminequivalent relative to $\mathcal{H B}^{n}(A, B)$.

Let us first assume that $\forall Y \exists X \varphi$ is false. Then, there is $I \subseteq Y$ such that for every $J \subseteq X, I \cup J \forall \forall \varphi$. Let $N=\{g\} \cup I \cup(Y \backslash I)^{\prime} \cup X \cup X^{\prime}$. We have that $N \models Q$, and $T_{Q}(N)=N$. Thus, $N \backslash T_{Q}(N)=\emptyset \subseteq A$ and, consequently, $N \in \operatorname{Mod}_{A}(Q)$. Let $M=\left.N\right|_{A \cup B}$. By the definition, $\left.M \subseteq N\right|_{A \cup B}$. Thus, the conditions (1) and (3) for $(M, N) \in \operatorname{Mod}_{A}^{B}(Q)$ hold. Next, by the property of $I, N$ is a minimal model of $Q$. It follows that $(M, N)$ satisfies the conditions (2) and (4) for $(M, N) \in \operatorname{Mod}_{A}^{B}(Q)$. Finally, we have $N \backslash T_{Q}(N)=\emptyset \subseteq M$. Thus, the condition (5) for $(M, N) \in \operatorname{Mod}_{A}^{B}(Q)$ holds and so, $(M, N) \in \operatorname{Mod}_{A}^{B}(Q)$. We observe that $T_{P}(N)=N \backslash\{g\}$ and $T_{Q}(N)=N$. Since $g \in B$ and $g \in N,\left.T_{P}(N)\right|_{B} \neq\left. T_{Q}(N)\right|_{B}$ follows. Hence, by Theorem 4.6 (we recall that $(M, N) \in \operatorname{Mod}_{A}^{B}(Q)$ and so, $\left.(M, N) \in \operatorname{Mod}_{A}^{B}(p)\right), P$ and $Q$ are not suppmin-equivalent relative to $\mathcal{H B}^{n}(A, B)$.

Next, let us assume that $\forall Y \exists X \varphi$ is true. Let $(M, N) \in$ $\operatorname{Mod}_{A}^{B}(P)$. Let us assume that $N$ is of the type 2. Let $\{g\} \cup$ $I \cup(Y \backslash I)^{\prime} \cup X \cup X^{\prime}$, where $I \subseteq Y$, be the $W$-core of $N$. Since $\forall Y \exists X \varphi$ is true, there is $J \subseteq X$ such that $I \cup J \models \varphi$. We define $K=\{g\} \cup I \cup(Y \backslash I)^{\prime} \cup J \cup(Y \backslash J)^{\prime}$. Clearly, $K \models$ $P$. We have $K \subset N$ and $\left.K\right|_{A \cup B}=\{g\}=\left.N\right|_{A \cup B}$ (we recall that $(A \cup B) \cap(W \backslash\{g\}=\emptyset)$. Thus, by the condition (2) for $(M, N) \in \operatorname{Mod}_{A}^{B}(P), K \forall \forall P$, a contradiction.

It follows that $N$ is of type 1 . Consequently, $T_{P}(N)=$ $T_{Q}(N)$ and so, $\left.T_{P}(N)\right|_{B}=\left.T_{Q}(N)\right|_{B}$. By Theorem 4.6, $P$ and $Q$ are suppmin-equivalent relative to $\mathcal{H B}^{n}(A, B)$.

This theorem cannot be extended to a wider class of finite sets $A$ and $B$. Let $A \cap B=\emptyset$ and $P, Q$ be two normal programs such that $\operatorname{Mod}_{A}^{B}(P)=\operatorname{Mod}_{A}^{B}(Q)$. Let $(X, Y) \in$ $\operatorname{Mod}_{A}^{B}(P)$ and $\left.b \in T_{P}(Y)\right|_{B}$. Then $b \in Y$ (as $T_{P}(Y) \subseteq Y$ ) and $b \notin A$ (as $b \in B$ and $A \cap B=\emptyset$ ). Since $Y \in \operatorname{Mod}_{A}(Q)$, $Y \backslash T_{Q}(Y) \subseteq A$. It follows that $b \in T_{Q}(Y)$ and, as $b \in$ $B,\left.b \in T_{Q}(Y)\right|_{B}$. Thus, $\left.\left.T_{P}(Y)\right|_{B} \subseteq T_{Q}(Y)\right|_{B}$ and, by symmetry, $\left.T_{P}(Y)\right|_{B}=\left.T_{Q}(Y)\right|_{B}$. Consequently, $P$ and $Q$ are suppmin-equivalent.

\section{Discussion}

In this section, we discuss relations between the semantics of supported models and stable models in the context of hyperequivalence. We start with a comparison of the characterizations for the most important cases, strong and uniform equivalence. We then move on to highlight some interesting differences in the complexity.
First, let us compare characterizations of the notion of strong equivalence, that is, hyperequivalence under the supported-model and stable-model semantics relative to the class of all programs, $\mathcal{H B}^{d}(A t, A t)$. To avoid references to $\operatorname{sh}(P)$ and $\operatorname{sh}(Q)$, we limit our discussion to the case when $P$ and $Q$ are normal.

According to Corollary 3.3, normal programs $P$ and $Q$ are supp-equivalent in this sense if and only if

1. $P$ and $Q$ have the same models, and for every model $Y$ of $P, T_{P}(Y)=T_{Q}(Y)$.

We note that in this case suppmin-equivalence has the same characterization (cf. Corollary 4.8).

Turning attention to strong equivalence under the stablemodel semantics, we recall that, as shown in (Turner 2003), the notion can be characterized in terms of SE-models. A pair of interpretations $(X, Y)$ with $X \subseteq Y$ is an SE-model of a program $P$ if $Y \models P$ and $X \models P^{Y}$. Two programs are strongly equivalent under the stable-model semantics if and only if they have the same SE-models. A simple reformulation yields that $P$ and $Q$ are strongly equivalent in the stable-model setting if and only if

2. $P$ and $Q$ have the same models, and for every model $Y$ of $P, \operatorname{Mod}(P[Y])=\operatorname{Mod}(Q[Y])$,

where $P[Y]=P^{Y} \cup\{\leftarrow z \mid z \in A t \backslash Y\}, P^{Y}=\{h d(r) \leftarrow$ $\left.b d^{+}(r) \mid r \in P, Y \models b d^{-}(r)\right\}$ is the reduct of $P$ with respect to $Y$, and $\operatorname{Mod}(R)$ stand for the set of (classical) models of a program $R$.

Despite differences between the characterizations (1) and (2), the basic intuition is quite similar in both settings. First, one checks whether the candidate interpretations $Y$, that is, interpretations that might become a supported/stable model once a program is suitably extended, are the same for the two programs under consideration. In each case, these candidate interpretations $Y$ are models of a program. Then, one checks whether any such candidate interpretation has the same effect on both programs. In the case of the supportedmodel semantics, this effect of $Y$ on a program $R$ is measured by $T_{R}(Y)$ (and so, $T_{P}(Y)=T_{Q}(Y)$ is required), while in the case of the stable-model semantics, it is given by $\operatorname{Mod}(R[Y])$, the set of models of an "extended" reduct of $R$ with respet to $Y$ (and so, $\operatorname{Mod}(P[Y])=\operatorname{Mod}(Q[Y])$, must hold).

Next, we will compare characterizations of uniform equivalence under supported minimal and stable models (we recall that, by Theorem 3.2, in case of supported models, strong and uniform equivalence coincide). Our characterization of suppmin-equivalence uses the definition of $\operatorname{Mod}_{A}^{B}(P)$ as given in Section 4. This definition simplifies for uniform equivalence (that is, for $A=A t$ and $B=\emptyset$ ) as follows: $(X, Y) \in \operatorname{Mod}_{A t}^{\emptyset}(P)$ if and only if
1. $Y \models P$
2. $X \subseteq Y$
3. for each $Z$ with $X \subseteq Z \subset Y, Z \not \models P$
4. $Y \backslash T_{P}(Y) \subseteq X$. 
By Corollary 4.7, uniform suppmin-equivalence between programs $P$ and $Q$ holds if and only if $\operatorname{Mod}_{A t}^{\emptyset}(P)=$ $\operatorname{Mod}_{A t}^{\emptyset}(Q)$.

To characterize uniform equivalence for the case of stable models, (Eiter, Fink, \& Woltran 2007) introduced UEmodels as special SE-models. A pair $(X, Y)$ is an UE-model of $P$, if

1. $Y \models P$

2. $X \subseteq Y$

3. for each $Z$ with $X \subset Z \subset Y, Z \not \forall P^{Y}$

4. $X \models P^{Y}$.

Hence, in other words, UE-models of $P$ are all SE-models of $P$ of the form $(Y, Y)$ plus SE-models $(X, Y)$ of $P$, where $X$ is maximal among the proper subsets of $Y$ that can appear with $Y$ in an SE-model. Finite programs $P$ and $Q$ are uniformly equivalent with respect to stable models if and only if the UE-models of $P$ and $Q$ coincide (Eiter, Fink, \& Woltran 2007) (the case of uniform equivalence of infinite programs has a slightly more elaborate characterization).

We will now compare the two characterizations for finite programs. Again, we observe that in the suppmin model case, $T_{P}(Y)$ plays a major role, while in the stable case, this role is taken over by the reduct $P^{Y}$. However, the remaining parts of the characterization show interesting similarities. On the one hand, as already discussed above, $Y$ serves as a candidate to become a supported/stable model after some program extension. On the other hand, we observe that both characterizations depend on a very similar set of countermodels (either of the the program itself, or of the reduct $P^{Y}$ ) which are subsets of $Y$. For infinite programs, direct comparison of uniform equivalence under the two semantics gets harder since, as we noted, the UE-model characterization of uniform equivalence for stable-model semantics does not hold any more (see (Eiter, Fink, \& Woltran 2007) for details on this issue).

We now turn to the complexity results, where some interesting differences can be observed (the complexity results for the stable model semantics we discuss below are from (Eiter, Fink, \& Woltran 2007; Woltran 2008)): First, deciding hyperequivalence with respect to supported models is coNP-complete, no matter how the context $\mathcal{H B}(A, B)$ is specified, as shown in Section 5. The same complexity class captures deciding hyperequivalence under stable models, as long as we restrict to normal programs. However, for disjunctive logic programs, deciding hyperequivalence in the stable-semantics setting is more complex for most instantiations of $\mathcal{H B}(A, B)$ (one exception is the case of strong equivalence, that is, the case $A=B=A t$, which remains coNP-complete). On the other hand, for supported models, disjunctions do not play a major role, and thus deciding hyperequivalence with respect to supported models remains in coNP even for disjunctive programs.

Changing the semantics to suppmin models has a more substantial effect, as we have shown in Section 6. Indeed, the complexity of deciding hyperequivalence with respect to suppmin models goes up to $\Pi_{2}^{P}$-completeness (already for normal programs). A notable exception is the case when at

\begin{tabular}{|r|ccc|}
\hline normal / disjunctive & $\mathcal{H} \mathcal{B}(A t, A t)$ & $\mathcal{H B}(A t, \emptyset)$ & $\mathcal{H} \mathcal{B}(A, B)$ \\
\hline supp & $\mathrm{coNP} / \mathrm{coNP}$ & $\mathrm{coNP} / \operatorname{coNP}$ & $\mathrm{coNP} / \mathrm{coNP}$ \\
suppmin & coNP/coNP & coNP/coNP & $\Pi_{2}^{P} / \Pi_{2}^{P}$ \\
stable & coNP/coNP & $\operatorname{coNP} / \Pi_{2}^{P}$ & $\operatorname{coNP} / \Pi_{2}^{P}$ \\
\hline
\end{tabular}

Table 1: Complexity of hyperequivalence for different semantics.

least one of $A$ and $B$ consists of all atoms, for which the corresponding problems of deciding hyperequivalence remain in coNP. Interestingly, this is not necessarily so in the stable-semantics world. As mentioned above, this holds for strong equivalence ( $A=B=A t$ ), but uniform equivalence ( $A=A t, B=\emptyset$ ) with respect to stable models remains $\Pi_{2}^{P}$ complete for disjunctive programs, while uniform suppminequivalence, as we noted, drops back to coNP.

Table 1 highlights these results in terms of completeness results, comparing the case of normal and disjunctive programs with respect to the different semantics and different instantiation of the context class, including strong-, uniform-, and the general case of hyperequivalence.

\section{Conclusions}

In this paper we extended the concept of hyperequivalence to two other major semantics of logic programs: the supportedmodel semantics and the supported minimal model semantics. We characterized these concepts of hyperequivalence and derived several complexity results.

Our characterizations were mainly based on the (partial) one-step provability operator $T_{P}$ (van Emden \& Kowalski 1976) and thus, unlike in the case of stable-model semantics, did not require any references to the reduct. However, some similarities to the case of the stable-model semantics appeared for more complex versions of hyperequivalence we studied, namely relativized supp- and suppmin-equivalence, which required additional concepts such as sets $\operatorname{Mod}_{A}(P)$ and $\operatorname{Mod}_{A}^{B}(P)$.

As concerns the complexity, the picture is uniform in the case of hyperequivalence with respect to supported models - problems that arise naturally turn out to be coNPcomplete. The situation is different for hyperequivalence with respect to suppmin models. When at least one of the sets $A$ and $B$ consists of all atoms, the corresponding problems of deciding hyperequivalence are coNP-complete. As soon as this is not the case, the complexity goes up and the decision problems become $\Pi_{2}^{P}$-complete. The results we presented demonstrate that with problems in which the departure from $A=A t$ and $B=A t$ is major: $A$ and $B$ are required to be finite (either as a parameter of the problem, or a part of the input). However, in some cases a much less drastic change has the same effect on the complexity. For instance, one can show that for every finite $A, B \subseteq A t$ such that $A \neq \emptyset$, the following problem is $\Pi_{2}^{P}$-complete: given normal programs $P$ and $Q$, decide whether $P$ and $Q$ are suppmin-equivalent relative to $\mathcal{H B}^{n}(A t \backslash A, B)$. Thus, even if just one atom from $A t$ is forbidden from appearing in heads of rules in context programs, the complexity jumps 
one level up. For a detailed analysis of this behavior we refer to (Truszczyński \& Woltran 2008).

While to the best of our knowledge, this is the first paper concerning hyperequivalence for supported (minimal) semantics, hyperequivalence between programs with respect to other semantics have been studied extensively. The concept of uniform equivalence appeared first in the area of databases in the setting of DATALOG. In that setting queries are (non-ground) programs. Uniform equivalence of programs was introduced by (Sagiv 1988), as a decidable approximation to query equivalence, and thus as a tool for query optimization. Several other equivalence notions in that context were studied in (Maher 1988).

In the area of logic programming with the stable-model semantics, the need for stronger (than ordinary) equivalence was already recognized in (Brass \& Dix 1997; Inoue \& Sakama 1998; Lifschitz, Tang, \& Turner 1999), before (Lifschitz, Pearce, \& Valverde 2001) coined the name of strong equivalence for "equivalence for substitution." In particular, (Brass \& Dix 1997; Lifschitz, Tang, \& Turner 1999) defined local rule transformations which retained the semantics of entire programs and thus provided first explicit results in this area. Papers following (Lifschitz, Pearce, \& Valverde 2001) dealt with characterizations of strong equivalence (Lin 2002; Turner 2003; de Jongh \& Hendriks 2003), studied other forms of equivalence (Inoue \& Sakama 2004; Eiter, Tompits, \& Woltran 2005; Oikarinen \& Janhunen 2006; Oetsch, Tompits, \& Woltran 2007; Woltran 2008) or were concerned with programs transformations (Eiter, Fink, \& Woltran 2007; Eiter et al. 2006; Lin \& Chen 2007; Wong 2008).

We mentioned in the introduction that our work may have implications for other nonmonotonic logics, most notably, autoepistemic logic of Moore with the semantics of expansions and moderately grounded expansions. We will now discuss this issue in more detail. We recall that a normal logic program rule

$$
a \leftarrow b_{1}, \ldots, b_{m}, \operatorname{not} c_{1}, \ldots, n \text { not } c_{n},
$$

can be interpreted as a modal formula (called modal rule)

$$
K b_{1} \wedge \ldots \wedge K b_{m} \wedge \neg K c_{1} \wedge \ldots \wedge \neg K c_{n} \supset a .
$$

This interpretation was first proposed by Konolige (Konolige 1988). It is known (Marek \& Truszczyński 1993) that there is a precise correspondence between supported models (supported minimal models) of a normal program $P$ and expansions (moderately grounded expansions) of the modal interpretation of $P$ (the theory consisting of modal rules corresponding to rules in $P$ ).

By a modal program we mean a theory in the modal language that consists of modal rules. Let $A, B$ be two sets of atoms. We denote by $\mathcal{H B}^{m}(A, B)$ the set of all modal programs consisting of modal rules with the antecedent containing only atoms from $B$ and the consequent being an atom from $A$. Due to the correspondence discussed above (and under a natural extension of the one-step provability operator to the setting of modal programs), the characterizations of supp-equivalence and suppmin-equivalence of normal programs relative to $\mathcal{H B}^{n}(A, B)$ lift literally to hyperequivalence under expansions and moderately grounded ex- pansions of modal programs relative to modal programs in $\mathcal{H B}^{m}(A, B)$.

While concerning only theories of some restricted syntactic form, these characterizations suggest that the hyperequivalence in autoepistemic logic could be treated in full generality. The fact that most arguments in this paper have a strong algebraic flavor and thus may only loosely depend on specific syntactic features of logic programs adds further credibility to that contention. In our future work, we will aim to develop algebraic generalizations of the characterizations presented in this paper (algebraic generalizations of hyperequivalence under the stable-model semantics were developed in (Truszczynski 2006)), and we will study hyperequivalence in autoepistemic logic without imposing syntactic restrictions on formulas.

Another interesting research direction concerns program simplification, for which our characterizations serve as a natural starting point. Moreover, in combination with the aforementioned extensions to autoepistemic logic, such techniques might also help to study new normal-form translations within that logic.

\section{References}

Apt, K. 1990. Logic Programming. In van Leeuven, J., ed., Handbook of Theoretical Computer Science. Elsevier. 493-574.

Brass, S., and Dix, J. 1997. Characterizations of the Disjunctive Stable Semantics by Partial Evaluation. Journal of Logic Programming 32(3):207-228.

Cabalar, P.; Odintsov, S.; Pearce, D.; and Valverde, A. 2006. Analysing and Extending Well-Founded and Partial Stable Semantics Using Partial Equilibrium Logic. In Etalle, S., and Truszczynski, M., eds., Proceedings of the 22nd International Conference on Logic Programming (ICLP 2006), volume 4079 of LNCS, 346-360. Springer.

Clark, K. 1978. Negation as Failure. In Gallaire, H., and Minker, J., eds., Logic and Data Bases. Plenum Press. 293322.

de Jongh, D., and Hendriks, L. 2003. Characterizations of Strongly Equivalent Logic Programs in Intermediate Logics. Theory and Practice of Logic Programming 3(3):259270.

Eiter, T., and Gottlob, G. 1992. Reasoning with Parsimonious and Moderately Grounded Expansions. Fundamenta Informaticae 17(1-2):31-53.

Eiter, T.; Fink, M.; Tompits, H.; Traxler, P.; and Woltran, S. 2006. Replacements in Non-Ground Answer-Set Programming. In Doherty, P.; Mylopoulos, J.; and Welty, C., eds., Proceedings of the 10th International Conference on Principles of Knowledge Representation and Reasoning (KR'06), 340-351. AAAI Press.

Eiter, T.; Fink, M.; and Woltran, S. 2007. Semantical Characterizations and Complexity of Equivalences in Answer Set Programming. ACM Transactions on Computational Logic 8(3). 53 pages.

Eiter, T.; Tompits, H.; and Woltran, S. 2005. On Solution Correspondences in Answer-Set Programming. In Pro- 
ceedings of the 19th International Joint Conference on Artificial Intelligence (IJCAI 2005), 97-102. Morgan Kaufmann.

Fages, F. 1994. Consistency of Clark's Completion and Existence of Stable Models. Journal of Methods of Logic in Computer Science 1:51-60.

Ferraris, P. 2005. On Modular Translations and Strong Equivalence. In Baral, C.; Greco, G.; Leone, N.; and Terracina, G., eds., Proceedings of the 8th International Conference on Logic Programming and Nonmonotonic Reasoning (LPNMR 2005), volume 3552 of LNCS, 79-91. Springer.

Gebser, M.; Schaub, T.; Tompits, H.; and Woltran, S. 2008. Alternative Characterizations for Program Equivalence under Answer-Set Semantics Based on Unfounded Sets. In Hartmann, S., and Kern-Isberner, G., eds., Foundations of Information and Knowledge Systems, 5th International Symposium, FoIKS 2008, Proceedings, volume 4932 of $L N C S$, 24-41. Springer.

Inoue, K., and Sakama, C. 1998. Negation as Failure in the Head. Journal of Logic Programming 35:39-78.

Inoue, K., and Sakama, C. 2004. Equivalence of Logic Programs Under Updates. In Proceedings of the 9th European Conference on Logics in Artificial Intelligence (JELIA 2004), volume 3229 of $L N C S, 174-186$. Springer.

Kaminski, M. 1991. Embedding a Default System into Nonmonotonic Logic. Fundamenta Informaticae 14(3):345-353.

Konolige, K. 1988. On the Relation Between Default and Autoepistemic Logic. Artificial Intelligence 35(3):343382.

Konolige, K. 1989. Errata: On the Relation between Default and Autoepistemic Logic. Artificial Intelligence 41(1): 115

Lee, J., and Lifschitz, V. 2003. Loop Formulas for Disjunctive Logic Programs. In Palamidessi, C., ed., Proceedings of the 19th International Conference on Logic Programming (ICLP 2003), volume 2916 of LNCS, 451-465. Springer.

Lifschitz, V.; Pearce, D.; and Valverde, A. 2001. Strongly Equivalent Logic Programs. ACM Transactions on Computational Logic 2(4):526-541.

Lifschitz, V.; Tang, L.; and Turner, H. 1999. Nested Expressions in Logic Programs. Annals of Mathematics and Artificial Intelligence 25(3-4):369-389.

Lin, F., and Chen, Y. 2007. Discovering Classes of Strongly Equivalent Logic Programs. Journal of Artificial Intelligence Research 28:431-451.

Lin, F., and Zhao, Y. 2002. ASSAT: Computing Answer Sets of a Logic Program by SAT Solvers. In Proceedings of the 18th National Conference on Artificial Intelligence (AAAI 2002), 112-117. AAAI Press.

Lin, F. 2002. Reducing Strong Equivalence of Logic Programs to Entailment in Classical Propositional Logic. In Fensel, D.; McGuinness, D.; and Williams, M., eds., Proceedings of the 8th International Conference on Principles of Knowledge Representation and Reasoning (KR 2002), 170-176. Morgan Kaufmann.

Maher, M. 1988. Equivalences of Logic Programs. In Minker, J., ed., Foundations of Deductive Databases and Logic Programming. Morgan Kaufmann. 627-658.

Marek, W., and Truszczyński, M. 1993. Nonmonotonic Logic; Context-Dependent Reasoning. Berlin: Springer.

Moore, R. 1985. Semantical considerations on nonmonotonic logic. Artificial Intelligence 25(1):75-94.

Oetsch, J.; Tompits, H.; and Woltran, S. 2007. Facts do not Cease to Exist Because They are Ignored: Relativised Uniform Equivalence with Answer-Set Projection. In Proceedings of the 22nd National Conference on Artificial Intelligence (AAAI 2007), 458-464. AAAI Press.

Oikarinen, E., and Janhunen, T. 2006. Modular Equivalence for Normal Logic Programs. In Proceedings of the 17th European Conference on Artificial Intelligence (ECAI 2006), 412-416. IOS Press.

Sagiv, Y. 1988. Optimising DATALOG Programs. In Minker, J., ed., Foundations of Deductive Databases and Logic Programming. Morgan Kaufmann. 659-698.

Truszczyński, M., and Woltran, S. 2008. Relativized hyperequivalence of logic programs for modular programming. In Proceedings of the 24th International Conference on Logic Programming (ICLP 2008), LNCS. Springer. To appear.

Truszczyński, M. 1991. Modal Nonmonotonic Logic with Restricted Application of the Negation as Failure to Prove Rule. Fundamenta Informaticae 14(3):355-366.

Truszczynski, M. 2006. Strong and Uniform Equivalence of Nonmonotonic Theories - An Algebraic Approach. Annals of Mathematics and Artificial Intelligence 48(3-4):245-265.

Turner, H. 2003. Strong Equivalence Made Easy: Nested Expressions and Weight Constraints. Theory and Practice of Logic Programming 3(4-5):609-622.

van Emden, M., and Kowalski, R. 1976. The Semantics of Predicate Logic as a Programming Language. Journal of the ACM 23(4):733-742.

Woltran, S. 2008. A Common View on Strong, Uniform, and Other Notions of Equivalence in Answer-Set Programming. Theory and Practice of Logic Programming $8(2): 217-234$.

Wong, K. 2008. Sound and Complete Inference Rules for SE-Consequence. Journal of Artificial Intelligence Research 31:205-216. 